\title{
A new Fast Multipole formulation for the elastodynamic half-space Green's tensor
}

\author{
Stéphanie Chaillat, Marc Bonnet \\ POEMS (UMR 7231 CNRS-ENSTA-INRIA), ENSTA Paristech, France \\ stephanie.chaillat@ensta.fr,marc.bonnet@ensta.fr
}

\begin{abstract}
In this article, a version of the frequency-domain elastodynamic Fast Multipole-Boundary Element Method (FM-BEM) for semi-infinite media, based on the half-space Green's tensor (and hence avoiding any discretization of the planar traction-free surface), is presented. The half-space Green's tensor is often used (in non-multipole form until now) for computing elastic wave propagation in the context of soil-structure interaction, with applications to seismology or civil engineering. However, unlike the fullspace Green's tensor, the elastodynamic half-space Green's tensor cannot be expressed using derivatives of the Helmholtz fundamental solution. As a result, multipole expansions of that tensor cannot be obtained directly from known expansions, and are instead derived here by means of a partial Fourier transform with respect to the spatial coordinates parallel to the free surface. The obtained formulation critically requires an efficient quadrature for the Fourier integral, whose integrand is both singular and oscillatory. Under these conditions, classical Gaussian quadratures would perform poorly, fail or require a large number of points. Instead, a version custom-tailored for the present needs of a methodology proposed by Rokhlin and coauthors, which generates generalized Gaussian quadrature rules for specific types of integrals, has been implemented. The accuracy and efficiency of the proposed formulation is demonstrated through numerical experiments on single-layer elastodynamic potentials involving up to about $N=6 \times 10^{5}$ degrees of freedom. In particular, a complexity significantly lower than that of the non-multipole version is shown to be achieved.
\end{abstract}

\section{Introduction}

The main advantage of the boundary element method (BEM) is that only the domain boundaries (and possibly interfaces) are discretized, leading to a reduction of the number of degrees of freedom (DOFs) relative to domain-discretization methods. Moreover, elastodyamic field equations and radiation conditions are exactly satisfied by the formulation [26], which avoids cumulative effects of grid dispersion and makes the BEM well suited for modeling wave propagation in unbounded domains. However, the standard BEM [3] leads to fully-populated matrices, which results in high computational complexity $\left(O\left(N^{2}\right)\right.$ per iteration using an iterative solver such as GMRES, where $N$ denotes the number of boundary degrees of freedom (DOFs) of the BE model) and severe problem size limitations induced by the $O\left(N^{2}\right)$ size of the influence matrix.

The advent of accelerated BE methodologies has dramatically improved the capabilities of BEMs for many areas of application, in a large part owing to the rapid development of the Fast Multipole Method (FMM) over the last 15-20 years [29]. Such approaches have resulted in considerable solution speedup, memory reduction and model size increase. The FMM inherently relies on iterative solvers (usually GMRES), so as to avoid actual computation and storage of the fully-populated influence matrix, and is known to require $O(N \log N)$ CPU time per iteration for Helmholtz-type equations [10,11]. Elastodynamic FMM formulations in the frequency domain have been investigated in e.g. [5] and [16] for the computation of seismic waves. The methodology of [16] has later been improved in [6] for homogeneous semi-infinite elastic propagation domains, through incorporation of recent advances of FMM implementations for Maxwell equations [10], allowing to run BEM models of size up to $N=O\left(10^{6}\right)$ on a single-processor PC. Other investigations address e.g. the time-domain elastodynamic FMM [35] or spatially periodic media [23]. 
All elastodynamic FMM formulations to date are based on the full-space Green's tensor. However, many practical situations of interest for modelling e.g. seismic waves or soil-structure interaction involve semi-infinite media. Existing formulations therefore entail truncated discretizations of the free surface. In such cases, both the potentially large number of additional DOFs required for the free surface and the selection of a suitable truncation radius are serious issues. An acceptable size of the meshed part of the free surface was empirically estimated in $[6,18]$ as about $3-5$ times the radius of the surface irregularity of interest. Assuming a uniform mesh density (with element sizes typically set to a fixed fraction, say $1 / 10$, of the shear wavelength), this results in $\mathrm{BE}$ model sizes of 10-15 times that of the meshed irregularity. To avoid meshing (part of) the free surface, one must resort to BEM formulations based on the half-space elastodynamic Green's tensor. The latter is known $[2,24]$ and applied in dynamic soil-structure interaction $[9,22]$ since some time; it is expressed in Fourier-Bessel integral form (involving oscillating integrals over infinite intervals), with no closed-form expression available. Its numerical evaluation is therefore complex and time-consuming [21,28]. Singularity and regularization issues for the half-space Green's tensor are addressed in [32], and also [1,30] for layered half-spaces, with applications to soil-structure interaction. Formulations based on full-space and half-space Green's tensors are compared in [31], the latter being found therein to entail prohibitive computational costs.

Unlike its full-space counterpart, multipole expansions of the elastodynamic half-space Green's tensor cannot be obtained directly as it cannot be expressed using derivatives of simpler kernels having known multipole expansions. This article addresses the resulting current lack of available accelerated BEMs based on the half-space Green's tensor. To this aim, the derivation of the latter is revisited, so as to recast it in a separated-variable form that enables fast computations. The obtained formulation critically requires an efficient quadrature for the resulting Fourier integral, whose integrand is both singular and oscillatory. Under these conditions, classical Gaussian quadratures would perform poorly, fail or require a large number of points. We adopt instead the methodology proposed by Rokhlin and coauthors $[4,8,36]$, which generates generalized Gaussian quadrature (GGQ) rules for specific types of integrals and is in particular useful for implementing some forms of the FMM. For instance, GGQ-generating algorithms have been developed in [36] and [8], respectively, for integrals involved in the low-frequency [19] and wideband [7] versions of the FMM for the 3D Helmholtz equation. The tabulated GGQ rules given

Box 1 Notations used in this article.

$\begin{array}{cl}\mu, \nu, \rho, \omega & \text { Shear modulus, Poisson's ratio, mass density, circular frequency } \\ \mathcal{C} & \text { Fourth-order elasticity tensor } \\ k_{\mathrm{P}}, k_{\mathrm{S}}, k_{\mathrm{R}} & \text { P, S and Rayleigh wavenumbers } \\ \kappa^{2} & :=\frac{1-2 \nu}{2(1-\nu)} \\ \text { prime symbol } & \text { denotes differentiation with respect to } y_{3} \\ \boldsymbol{a} \otimes \boldsymbol{b}, \boldsymbol{a} \cdot \boldsymbol{b}, \boldsymbol{A}: \boldsymbol{B} & \text { Tensor product, single inner product, double inner product } \\ \boldsymbol{S} & \left.:=\boldsymbol{I}-2 \boldsymbol{e}_{3} \otimes \boldsymbol{e}_{3} \text { (symmetry with respect to plane } y_{3}=0\right) \\ \boldsymbol{\xi} & :=\left(\xi_{1}, \xi_{2}\right) \text { transformed coordinates in the Fourier space } \\ \xi^{2} & :=\xi_{1}^{2}+\xi_{2}^{2} \\ s_{a}(\xi) & :=\sqrt{\xi^{2}-k_{a}^{2}} \quad(a=\mathrm{P}, \mathrm{S}) \\ \beta(\xi) & :=k_{\mathrm{S}}^{2}-2 \xi^{2}=-\left(s_{\mathrm{S}}^{2}(\xi)+\xi^{2}\right) \\ \delta(\xi) & :=\beta^{2}(\xi)-4 \xi^{2} s_{\mathrm{P}}(\xi) s_{\mathrm{S}}(\xi) \\ \boldsymbol{q}_{a}^{ \pm}(\boldsymbol{\xi}) & := \pm \mathrm{i} \boldsymbol{\xi}+s_{a}(\xi) \boldsymbol{e}_{3} \\ \boldsymbol{V}_{\mathrm{S}}^{ \pm}(\boldsymbol{\xi}) & := \pm\left[s_{\mathrm{S}}(\xi) \boldsymbol{q}_{\mathrm{S}}^{ \pm}(\boldsymbol{\xi})+k_{\mathrm{S}}^{2} \boldsymbol{e}_{3}\right] \\ \boldsymbol{V}_{\mathrm{P}}^{ \pm}(\boldsymbol{\xi}) & := \pm s_{\mathrm{P}}^{-1}(\xi) \boldsymbol{q}_{\mathrm{P}}^{ \pm}(\boldsymbol{\xi}) \\ p, r & \text { Size of initial Gauss-Legendre rule; rank of discretized input functions } \\ n_{\xi}, n_{\alpha} & \text { Sizes of final (generalized Gaussian) radial rule and of angular rule }\end{array}$


in $[4,27,36]$ cannot be used directly here because the integrals at hand involve a Rayleigh pole, whereas corresponding representations for Helmholtz problems do not. Instead, the GGQ generation methodology described in [4] is specialized to, and implemented for, the present needs. The accuracy and efficiency of the resulting formulation are then demonstrated through numerical experiments on integral operators involving up to about $N=6 \times 10^{5}$ DOFs, with emphasis on the behavior of the GGQ rules generated. In particular, a complexity significantly lower than that of the non-multipole version of the half-space Green's tensor is empirically shown to be achieved. This investigation thus lays a suitable groundwork for a forthcoming full implementation of elastodynamic fast BEMs based on the half-space Green's tensor.

The article is organized as follows. After reviewing relevant BIE formulations in Sec. 2, the proposed FMM-compatible formulation of the half-space Green's tensor is presented in Sec. 3. An overview of the resulting fast method is given, and the main quadrature issues are reviewed, in Sec. 4. The methodology for generating the required GGQ rules is described in Sec. 5. Finally, numerical tests are reported and discussed in Sec. 6 .

Notations. In Box 1, notations used throughout this Article are summarized. Roman letters denote continuous scalar fields and operators, bold letters denote vectors.

\section{Standard boundary integral equation}

In this section, relevant continuous BIE formulations for the propagation of elastic waves in semiinfinite homogeneous regions that may feature topographic irregularities or buried objects are reviewed. Accordingly, letting $\Omega_{F}$ denote the half-space $\left\{\boldsymbol{y}=\left(y_{1}, y_{2}, y_{3}\right) \mid y_{3}<0\right\}$ bounded by the infinite traction-free planar surface $\Gamma_{F}=\left\{\boldsymbol{y} \mid y_{3}=0\right\}$, configurations of interest in this article are semi-infinite domains $\Omega$ which deviate from the half-space $\Omega_{F}$ (e.g. because of surface irregularities) only in a region of finite size. The boundary $\partial \Omega$ is thus of the form $\partial \Omega=S \cup \Gamma_{t} \cup \Gamma_{0}$, where the bounded (and possibly non-connected) surface $S:=\partial \Omega \backslash\left(\partial \Omega \cap \Gamma_{F}\right)$ defines topographic irregularities or buried obstacles, the bounded portion $\Gamma_{t}$ of $\Gamma_{F}$ supports prescribed tractions, and $\Gamma_{0} \subset \Gamma_{F}$ is the unbounded planar component of the traction-free surface (Fig. 1). Boundary conditions on $S$, which are necessary for the elastodynamic problem to be well-posed, are left unspecified. The propagation medium occupying $\Omega$ is an homogeneous isotropic elastic solid material characterized by its shear modulus $\mu$, Poisson's ratio $\nu$ and mass density $\rho$. Time-harmonic motions with circular frequency $\omega$ are assumed, and the implicit factor $e^{-i \omega t}$ will as usual be omitted. Cauchy's first law for time-harmonic motions relates the displacement $\boldsymbol{u}$ and the stress tensor $\boldsymbol{\sigma}$ through:

$$
\boldsymbol{\nabla} \cdot \boldsymbol{\sigma}+\rho \omega^{2} \boldsymbol{u}=-\rho \boldsymbol{b} \quad \text { in } \Omega,
$$

where $\rho \boldsymbol{b}$ is a given body force density. The isotropic linear elastic constitutive equation, expressed in terms of the fourth-order elasticity tensor $\mathcal{C}$, reads

$$
\boldsymbol{\sigma}=\mathcal{C}: \boldsymbol{\nabla} \boldsymbol{u}, \quad \mathcal{C}_{i j k \ell}=\mu\left[\frac{2 \nu}{1-2 \nu} \delta_{i j} \delta_{k \ell}+\delta_{i k} \delta_{j \ell}+\delta_{j k} \delta_{i \ell}\right]
$$

where $\delta_{i j}$ is the Kronecker symbol. In (1), (2) and thereafter, the symbols "." and ":" respectively denote simple and double tensor inner products. Combining (1) and (2) yields the well-known second order Navier equation for $\boldsymbol{u}$ [14].

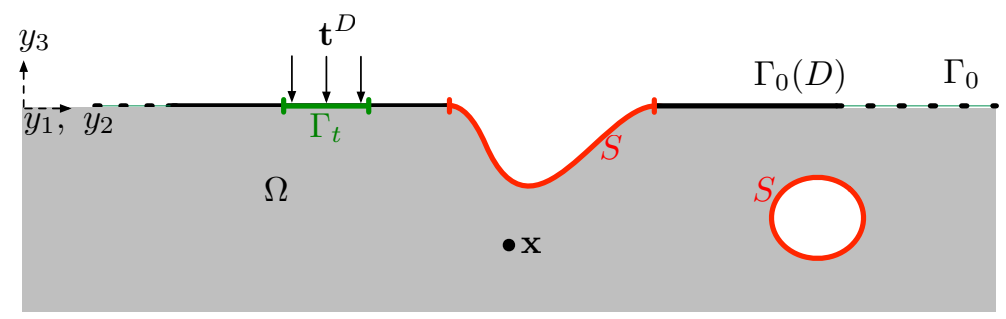

Figure 1: Geometry and notations. 
BIE based on the full-space elastodynamic Green's tensor. Assuming the absence of body forces, the displacement $\boldsymbol{u}$ is given at an interior point $\boldsymbol{x} \in \Omega$ by the well-known integral representation formula:

$$
\begin{aligned}
\boldsymbol{u}(\boldsymbol{x})=\int_{S} \boldsymbol{t}(\boldsymbol{y}) \cdot \boldsymbol{U}_{\infty}(\boldsymbol{x}, \boldsymbol{y} ; \omega) \mathrm{d} S_{y}+\int_{\Gamma_{t}} \boldsymbol{t}^{\mathrm{D}}(\boldsymbol{y}) \cdot \boldsymbol{U}_{\infty}(\boldsymbol{x}, \boldsymbol{y} ; \omega) \mathrm{d} S_{y} & \\
& -\int_{S \cup \Gamma_{t} \cup \Gamma_{0}} \boldsymbol{u}(\boldsymbol{y}) \cdot \boldsymbol{T}_{\infty}(\boldsymbol{x}, \boldsymbol{y} ; \omega) \mathrm{d} S_{y} \quad(\boldsymbol{x} \in \Omega)
\end{aligned}
$$

where $\boldsymbol{t}:=\boldsymbol{n} \cdot \boldsymbol{\sigma}$ is the traction vector on $S$ and $\boldsymbol{t}^{\mathrm{D}}$ the prescribed value of the traction on $\Gamma_{t}$; moreover, $\boldsymbol{U}_{\infty}(\boldsymbol{x}, \boldsymbol{y} ; \omega)$ and $\boldsymbol{T}_{\infty}(\boldsymbol{x}, \boldsymbol{y} ; \omega)$ denote the elastodynamic Green's tensor, defined such that $\boldsymbol{U}_{\infty} \cdot \boldsymbol{F}$ and $\boldsymbol{T}_{\infty} \cdot \boldsymbol{F}$ are the displacement and traction vector, respectively, generated at any point $\boldsymbol{y} \in \mathbb{R}^{3}$ of the full space by a unit point force $\boldsymbol{F}$ applied at $\boldsymbol{x} \in \mathbb{R}^{3}$.

Well-known results on the limiting values of elastic potentials as the collocation point $\boldsymbol{x}$ reaches the boundary $[20,25]$ allow to deduce from (3) the boundary integral equation

$$
\begin{aligned}
\boldsymbol{c}(\boldsymbol{x}) \cdot \boldsymbol{u}(\boldsymbol{x})+(\mathrm{P} . \mathrm{V} .) \int_{S \cup \Gamma_{t} \cup \Gamma_{0}} \boldsymbol{u}(\boldsymbol{y}) \cdot \boldsymbol{T}_{\infty}(\boldsymbol{x}, \boldsymbol{y} ; \omega) \mathrm{d} S_{y}-\int_{S} \boldsymbol{t}(\boldsymbol{y}) \cdot \boldsymbol{U}_{\infty}(\boldsymbol{x}, \boldsymbol{y} ; \omega) \mathrm{d} S_{y} \\
\quad=\int_{\Gamma_{t}} \boldsymbol{t}^{\mathrm{D}}(\boldsymbol{y}) \cdot \boldsymbol{U}_{\infty}(\boldsymbol{x}, \boldsymbol{y} ; \omega) \mathrm{d} S_{y} \quad\left(\boldsymbol{x} \in S \cup \Gamma_{t} \cup \Gamma_{0}\right) .
\end{aligned}
$$

Due to its strong singularity for $\boldsymbol{y}=\boldsymbol{x}$, integrals of $\boldsymbol{T}_{\infty}$ are defined only in the Cauchy principal value (CPV) sense (as indicated by the notation P.V.) while a free-term $\boldsymbol{c}(\boldsymbol{x})$ arises. The latter is equal to $0.5 \boldsymbol{I}$ (with $\boldsymbol{I}$ denoting the second-order identity tensor) in the usual case where $\partial \Omega$ is smooth at $\boldsymbol{x}$, and is otherwise a known (second-order tensor-valued) function of the local geometry of $\partial \Omega$ at $\boldsymbol{x}$. Note that additional information in the form of boundary conditions on $S$ must be specified to make integral equation (4) well-posed.

BIE based on the half-space elastodynamic Green's tensor. For the geometrical configurations considered in this article, the first integral operator in (4) involves an integral over the unbounded surface $\Gamma_{0}$. Practical implementation of (4) thus requires a truncated version $\Gamma_{0}(D)$ of the traction-free surface $\Gamma_{0}$, e.g. bounded by a circle of radius $D$. As the truncation distance $D$ must be sufficiently large relative to the diameter of the irregularity $S$, the BE discretization of $\Gamma_{0}(D)$ usually introduces large numbers of DOFs, with obvious adverse effects on both computational speed and memory requirements.

The contribution of $\Gamma_{0}$ to equation (4) can be removed (and truncation issues avoided) by using the half-space Green's tensor $\boldsymbol{U}^{\mathrm{HS}}, \boldsymbol{T}^{\mathrm{HS}}$ [32], which satisfies the field equation (1) in the half-space $\Omega_{F}$ and the traction-free condition

$$
\boldsymbol{T}^{\mathrm{HS}}(\boldsymbol{x}, \boldsymbol{y} ; \omega):=\boldsymbol{e}_{3} \cdot \mathcal{C}: \nabla_{y} \boldsymbol{U}^{\mathrm{HS}}(\boldsymbol{x}, \boldsymbol{y} ; \omega)=\mathbf{0} \quad\left(\boldsymbol{y} \in \Gamma_{F}\right)
$$

on the planar surface $\Gamma_{F}$, rather than the full-space Green's tensor (see Sec. 3 for details on $\boldsymbol{U}^{\mathrm{HS}}$ ). In (5) and thereafter, the gradient operator ${ }^{\prime} \nabla^{\prime}$ conventionally adds one tensorial order from the left, i.e. (for example) $(\boldsymbol{\nabla} \boldsymbol{U})_{k i j}=\partial_{k} U_{i j}$. The integral equation (4) then takes the form (with collocation now needed on $S$ only)

$$
\begin{aligned}
\boldsymbol{c}(\boldsymbol{x}) \cdot \boldsymbol{u}(\boldsymbol{x})+(\mathrm{P} . \mathrm{V} .) \int_{S} \boldsymbol{u}(\boldsymbol{y}) \cdot \boldsymbol{T}^{\mathrm{HS}}(\boldsymbol{x}, \boldsymbol{y} ; \omega) \mathrm{d} S_{y}-\int_{S} \boldsymbol{t}(\boldsymbol{y}) \cdot \boldsymbol{U}^{\mathrm{HS}}(\boldsymbol{x}, \boldsymbol{y} ; \omega) \mathrm{d} S_{y} & \\
& =\int_{\Gamma_{t}} \boldsymbol{t}^{\mathrm{D}}(\boldsymbol{y}) \cdot \boldsymbol{U}^{\mathrm{HS}}(\boldsymbol{x}, \boldsymbol{y} ; \omega) \mathrm{d} S_{y} \quad(\boldsymbol{x} \in S) .
\end{aligned}
$$


Applying iterative linear solvers, such as GMRES [34], to the integral equation (6) essentially entails the evaluation of single-layer and double-layer elastic potentials of the form

$$
\mathcal{S}[\boldsymbol{t}](\boldsymbol{x}):=\int_{S} \boldsymbol{t}(\boldsymbol{y}) \cdot \boldsymbol{U}^{\mathrm{HS}}(\boldsymbol{x}, \boldsymbol{y} ; \omega) \mathrm{d} S_{y}, \quad \mathcal{D}[\boldsymbol{u}](\boldsymbol{x}):=\int_{S} \boldsymbol{u}(\boldsymbol{y}) \cdot \boldsymbol{T}^{\mathrm{HS}}(\boldsymbol{x}, \boldsymbol{y} ; \omega) \mathrm{d} S_{y}
$$

for given densities $\boldsymbol{t}, \boldsymbol{u}$. This article will focus on the single-layer potential $\mathcal{S}[\boldsymbol{t}]$, whose fast evaluation requires a multipole-type expansion for $\boldsymbol{U}^{\mathrm{HS}}$, as a representative computational task involved in a fast-BEM solution strategy; in particular, the case of $\mathcal{D}[\boldsymbol{u}]$ then follows with straightforward modifications.

In practice, potentials such as $\mathcal{S}[\boldsymbol{t}](\boldsymbol{x})$ need to be evaluated for a finite (large) set $\mathcal{X}$ of locations $\boldsymbol{x}$, while the boundary element discretization of $S$ reduces integrals to weighted sums over a finite (also large) set $\mathcal{Y}$ of quadrature points $\boldsymbol{y}$. The set $\mathcal{X}$ typically gathers either collocation nodes (when using collocation BIE formulations such as (6)) or quadrature nodes (when using Galerkin BIE for-

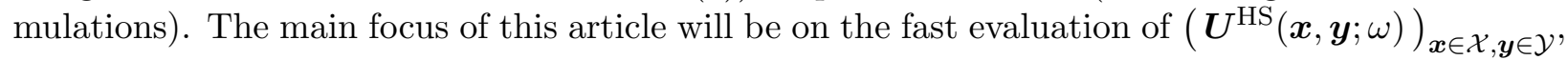
and of weighted sums over $\mathcal{Y}$, for large finite sets $\mathcal{X}, \mathcal{Y}$. Upon adaptation to $\mathcal{D}[\boldsymbol{u}]$ and in some cases to potentials involving hypersingular kernels, the proposed treatment is thus applicable not only to the BIE (6) but also to more-complex BIEs (e.g. formulations of Burton-Miller type) and to Galerkin BIE formulations.

\section{Multipole expansion for the elastodynamic half-space Green's tensor}

Our starting point for deriving a multipole expansion of $\boldsymbol{U}^{\mathrm{HS}}$ is the additive decomposition

$$
\boldsymbol{U}^{\mathrm{HS}}(\boldsymbol{x}, \boldsymbol{y} ; \omega)=\boldsymbol{U}_{\infty}(\boldsymbol{x}, \boldsymbol{y} ; \omega)+\overline{\boldsymbol{U}}_{\infty}(\boldsymbol{x}, \boldsymbol{y} ; \omega)+\boldsymbol{U}_{\mathrm{C}}(\boldsymbol{x}, \boldsymbol{y} ; \omega),
$$

where $\boldsymbol{U}_{\infty}$ is the elastic full-space Green's tensor and $\overline{\boldsymbol{U}}_{\infty}$, given by

$$
\overline{\boldsymbol{U}}_{\infty}(\boldsymbol{x}, \boldsymbol{y} ; \omega):=\boldsymbol{U}_{\infty}(\boldsymbol{S} \cdot \boldsymbol{x}, \boldsymbol{y} ; \omega) \cdot \boldsymbol{S},
$$

(with $\boldsymbol{S}:=\boldsymbol{I}-2 \boldsymbol{e}_{3} \otimes \boldsymbol{e}_{3}$ denoting the tensor associated with the symmetry with respect to the free surface $\left.y_{3}=0\right)$ is the image full-space Green's tensor, i.e. the value at $\boldsymbol{y}$ of the full-space Green's tensor for an image point force $\boldsymbol{S} \cdot \boldsymbol{F}$ applied at the mirror image source point $\boldsymbol{S} \cdot \boldsymbol{x}$ (Fig. 2). The complementary Green's tensor $\boldsymbol{U}_{\mathrm{C}}$ must therefore solve the homogeneous field equation (1) in $\Omega_{F}$ and be such that $\boldsymbol{U}^{\mathrm{HS}}$ given by (8) satisfies the free-surface condition (5) on $\Gamma_{F}$. The single-layer potential $\mathcal{S}[\boldsymbol{t}]$ can accordingly be given, using obvious notation, as the additive decomposition

$$
\mathcal{S}[\boldsymbol{t}](\boldsymbol{x})=\mathcal{S}_{\infty}[\boldsymbol{t}](\boldsymbol{x})+\overline{\mathcal{S}}_{\infty}[\boldsymbol{t}](\boldsymbol{x})+\mathcal{S}_{\mathrm{C}}[\boldsymbol{t}](\boldsymbol{x}) .
$$

A FMM treatment of integral equation (6) thus may exploit the "standard" FMM associated with the diagonal form-based decomposition of the full-space Green's tensor [6], for the evaluation of contributions $\mathcal{S}_{\infty}[\boldsymbol{t}](\boldsymbol{x}), \overline{\mathcal{S}}_{\infty}[\boldsymbol{t}](\boldsymbol{x})$. Attention is therefore focused on fast evaluation of the contribution $\mathcal{S}_{\mathrm{C}}[\boldsymbol{t}](\boldsymbol{x})$ involving the complementary Green's tensor.

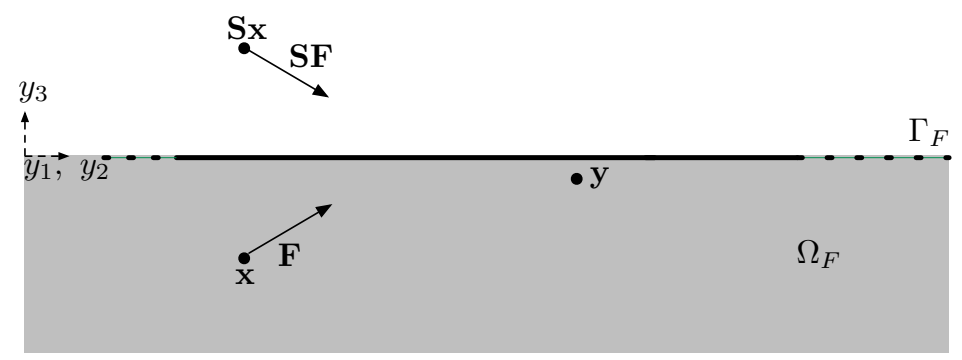

Figure 2: Notations for the definition of the image full-space Green's tensor. 


\subsection{Derivation in the Fourier space}

The proposed method for formulating the complementary Green's tensor is based on a partial Fourier transform with respect to the spatial coordinates $y_{1}, y_{2}$ parallel to the free surface $\Gamma_{F}$ (see Fig. 2), and therefore bears similarities with the method used for defining low-frequency FMMs [11, 19]. The partial Fourier transform $\hat{\boldsymbol{u}}$ of a generic scalar, vector or tensor field $\boldsymbol{u}$ is defined by

$$
\hat{\boldsymbol{u}}\left(\boldsymbol{\xi}, y_{3}\right)=\frac{1}{4 \pi^{2}} \int_{\mathbb{R}^{2}} e^{-\mathrm{i}\left(\xi_{1} y_{1}+\xi_{2} y_{2}\right)} \boldsymbol{u}\left(y_{1}, y_{2}, y_{3}\right) \mathrm{d} y_{1} \mathrm{~d} y_{2},
$$

where $\boldsymbol{\xi}:=\left(\xi_{1}, \xi_{2}\right)$ are the transformed coordinates associated with $\left(y_{1}, y_{2}\right)$. Using the prime $\left(^{\prime}\right)$ symbol to denote partial derivatives with respect to the vertical coordinate $y_{3}$, one then has

$$
\widehat{\nabla \boldsymbol{u}}\left(\boldsymbol{\xi}, y_{3}\right)=\mathrm{i} \boldsymbol{\xi} \otimes \hat{\boldsymbol{u}}\left(\boldsymbol{\xi}, y_{3}\right)+\boldsymbol{e}_{3} \otimes \hat{\boldsymbol{u}}^{\prime}\left(\boldsymbol{\xi}, y_{3}\right) .
$$

Full-space Green's tensor. While not necessary for the actual computation of the contribution $\mathcal{S}_{\infty}[\boldsymbol{t}](\boldsymbol{x})$, the partial Fourier transform $\hat{\boldsymbol{U}}_{\infty}$ of the full-space Green's tensor $\boldsymbol{U}_{\infty}$ and its image counterpart $\hat{\bar{U}}_{\infty}$ are needed for formulating the boundary condition $\boldsymbol{T}^{\mathrm{HS}}=\mathbf{0}$ on $\Gamma_{F}$ in partialFourier form (which implies that only the values at $y_{3}=0$ of $\hat{\boldsymbol{U}}_{\infty}, \hat{\boldsymbol{U}}_{\infty}$ and $\hat{\boldsymbol{U}}_{\infty}^{\prime}, \hat{\overline{\boldsymbol{U}}}_{\infty}^{\prime}$ are necessary). The tensor $\boldsymbol{U}_{\infty}$ is given by ([14], eq. 5.16.23):

$$
\boldsymbol{U}_{\infty}(\boldsymbol{x}, \boldsymbol{y} ; \omega)=\frac{1}{4 \pi k_{\mathrm{S}}^{2} \mu}\left[\nabla \nabla\left\{G\left(|\boldsymbol{y}-\boldsymbol{x}| ; k_{\mathrm{S}}\right)-G\left(|\boldsymbol{y}-\boldsymbol{x}| ; k_{\mathrm{P}}\right)\right\}-\Delta G\left(|\boldsymbol{y}-\boldsymbol{x}| ; k_{\mathrm{S}}\right) \boldsymbol{I}\right]
$$

where $G(r ; k)$ is the full-space fundamental solution for the Helmholtz equation with wavenumber $k$ :

$$
G(r ; k)=\frac{\exp (i k r)}{r}
$$

and $k_{\mathrm{P}}, k_{\mathrm{S}}$ are the wavenumbers corresponding to dilatational and shear elastic waves, i.e.:

$$
k_{\mathrm{S}}=\omega \sqrt{\rho / \mu}, \quad k_{\mathrm{P}}=\kappa k_{\mathrm{S}}, \quad \text { with } \quad \kappa^{2}:=\frac{1-2 \nu}{2(1-\nu)} .
$$

The partial Fourier transform $\hat{G}$ of $G(r ; k)$ is known [19]; it is given by

$$
\begin{aligned}
\hat{G}\left(\boldsymbol{\xi}, y_{3} ; \boldsymbol{x}, k_{a}\right) & =\frac{1}{2 \pi s_{a}} e^{\boldsymbol{q}_{a}^{-}(\boldsymbol{\xi}) \cdot \boldsymbol{x}} e^{-s_{a}(\xi) y_{3}} & \left(y_{3} \geq x_{3}\right), \quad a=\mathrm{P}, \mathrm{S}, \\
& =\frac{1}{2 \pi s_{a}} e^{-\boldsymbol{q}_{a}^{+}(\boldsymbol{\xi}) \cdot \boldsymbol{x}} e^{s_{a}(\xi) y_{3}} & \left(y_{3} \leq x_{3}\right), \quad a=\mathrm{P}, \mathrm{S},
\end{aligned}
$$

with $s_{a}(\xi):=\sqrt{\xi^{2}-k_{a}^{2}}, \xi^{2}=\|\boldsymbol{\xi}\|^{2}$ and having set

$$
\boldsymbol{q}_{a}^{-}(\boldsymbol{\xi}):=-\mathrm{i} \boldsymbol{\xi}+s_{a}(\xi) \boldsymbol{e}_{3}, \quad \boldsymbol{q}_{a}^{+}(\boldsymbol{\xi}):=\mathrm{i} \boldsymbol{\xi}+s_{a}(\xi) \boldsymbol{e}_{3} \quad(a=\mathrm{P}, \mathrm{S}) .
$$

One notes for later reference that applying (12) to (16a,b) yields

$$
\begin{aligned}
\widehat{\nabla G}\left(\boldsymbol{\xi}, y_{3} ; \boldsymbol{x}, k_{a}\right) & =-\hat{G}\left(\boldsymbol{\xi}, y_{3} ; \boldsymbol{x}, k_{a}\right) \boldsymbol{q}_{a}^{-}(\boldsymbol{\xi}) & & \left(y_{3} \geq x_{3}\right), \quad a=\mathrm{P}, \mathrm{S}, \\
& =\hat{G}\left(\boldsymbol{\xi}, y_{3} ; \boldsymbol{x}, k_{a}\right) \boldsymbol{q}_{a}^{+}(\boldsymbol{\xi}) & & \left(y_{3} \leq x_{3}\right), \quad a=\mathrm{P}, \mathrm{S} .
\end{aligned}
$$

Original source point. This case corresponds to $\boldsymbol{x} \in \Omega_{F}$, for which one has $y_{3}>x_{3}$ since only the case $y_{3}=0$ needs to be considered for enforcing (5). Taking the partial Fourier transform of (13) and exploiting (16a) and (18a) therein, one arrives at

$$
\hat{\boldsymbol{U}}_{\infty}\left(\boldsymbol{\xi}, y_{3} ; \boldsymbol{x}, \omega\right)=\hat{\boldsymbol{U}}_{\mathrm{P}}(\boldsymbol{\xi}) e^{\boldsymbol{q}_{\mathrm{P}}^{-}(\boldsymbol{\xi}) \cdot \boldsymbol{x}} e^{-s_{\mathrm{P}}(\xi) y_{3}}+\hat{\boldsymbol{U}}_{\mathrm{S}}(\boldsymbol{\xi}) e^{\boldsymbol{q}_{\mathrm{S}}^{-}(\boldsymbol{\xi}) \cdot \boldsymbol{x}} e^{-s_{\mathrm{S}}(\xi) y_{3}}
$$

with

$$
\hat{\boldsymbol{U}}_{\mathrm{P}}(\boldsymbol{\xi})=\frac{-1}{8 \pi^{2} \mu k_{\mathrm{S}}^{2} s_{\mathrm{P}}(\xi)} \boldsymbol{q}_{\mathrm{P}}^{-}(\boldsymbol{\xi}) \otimes \boldsymbol{q}_{\mathrm{P}}^{-}(\boldsymbol{\xi}), \quad \hat{\boldsymbol{U}}_{\mathrm{S}}(\boldsymbol{\xi})=\frac{1}{8 \pi^{2} \mu k_{\mathrm{S}}^{2} s_{\mathrm{S}}(\xi)}\left[\boldsymbol{q}_{\mathrm{S}}^{-}(\boldsymbol{\xi}) \otimes \boldsymbol{q}_{\mathrm{S}}^{-}(\boldsymbol{\xi})+k_{\mathrm{S}}^{2} \boldsymbol{I}\right] .
$$

Then, the traction Green's tensor $\boldsymbol{T}_{\infty}:=\boldsymbol{e}_{3} \cdot \mathcal{C}: \nabla_{y} \boldsymbol{U}_{\infty}$ is obtained in Fourier form as

$$
\hat{\boldsymbol{T}}_{\infty}(\boldsymbol{\xi})=\boldsymbol{D}(\boldsymbol{\xi}) \cdot \hat{\boldsymbol{U}}_{\infty}(\boldsymbol{\xi}, 0)+\boldsymbol{C} \cdot \hat{\boldsymbol{U}}_{\infty}^{\prime}(\boldsymbol{\xi}, 0)
$$


having used (12) and with

$$
\boldsymbol{C}=\mu\left[\boldsymbol{I}+\left(\kappa^{-2}-1\right) \boldsymbol{e}_{3} \otimes \boldsymbol{e}_{3}\right], \quad \boldsymbol{D}(\boldsymbol{\xi})=\mathrm{i} \mu\left[\boldsymbol{\xi} \otimes \boldsymbol{e}_{3}+\left(\kappa^{-2}-2\right) \boldsymbol{e}_{3} \otimes \boldsymbol{\xi}\right]
$$

(with $\kappa$ as defined in (15)). Inserting (19) in (20), one obtains

$$
\hat{\boldsymbol{T}}_{\infty}(\boldsymbol{\xi})=\left(\boldsymbol{D}(\boldsymbol{\xi})-s_{\mathrm{P}}(\xi) \boldsymbol{C}\right) \cdot \hat{\boldsymbol{U}}_{\mathrm{P}}(\boldsymbol{\xi}) e^{\boldsymbol{q}_{\mathrm{P}}^{-}(\boldsymbol{\xi}) \cdot \boldsymbol{x}}+\left(\boldsymbol{D}(\boldsymbol{\xi})-s_{\mathrm{S}}(\xi) \boldsymbol{C}\right) \cdot \hat{\boldsymbol{U}}_{\mathrm{S}}(\boldsymbol{\xi}) e^{\boldsymbol{q}_{\mathrm{S}}^{-}(\boldsymbol{\xi}) \cdot \boldsymbol{x}} .
$$

The following explicit expression of $\hat{\boldsymbol{T}}_{\infty}$ is then readily obtained by using the previously-given definitions of $\boldsymbol{C}, \boldsymbol{D}$ and $\hat{\boldsymbol{U}}_{\mathrm{P}}, \hat{\boldsymbol{U}}_{\mathrm{S}}$ :

$$
\begin{aligned}
\hat{\boldsymbol{T}}_{\infty}(\boldsymbol{\xi})=\frac{1}{8 \pi^{2} k_{\mathrm{S}}^{2}}\left\{\left[2 \boldsymbol{q}_{\mathrm{P}}^{-}(\boldsymbol{\xi}) \otimes \boldsymbol{q}_{\mathrm{P}}^{-}(\boldsymbol{\xi})+\left(2 \kappa^{2}-1\right) k_{\mathrm{S}}^{2} s_{\mathrm{P}}^{-1}(\xi) \boldsymbol{e}_{3} \otimes \boldsymbol{q}_{\mathrm{P}}^{-}(\boldsymbol{\xi})\right] e^{\boldsymbol{q}_{\mathrm{P}}^{-}(\boldsymbol{\xi}) \cdot \boldsymbol{x}}\right. & \\
- & {\left.\left[2 \boldsymbol{q}_{\mathrm{S}}^{-}(\boldsymbol{\xi}) \otimes \boldsymbol{q}_{\mathrm{S}}^{-}(\boldsymbol{\xi})+k_{\mathrm{S}}^{2}\left(\boldsymbol{I}+s_{\mathrm{S}}^{-1}(\xi) \boldsymbol{q}_{\mathrm{S}}^{-}(\boldsymbol{\xi}) \otimes \boldsymbol{e}_{3}\right)\right] e^{\boldsymbol{q}_{\mathrm{S}}^{-}(\boldsymbol{\xi}) \cdot \boldsymbol{x}}\right\} . }
\end{aligned}
$$

Image source point. This case corresponds to the choice $\boldsymbol{S} \cdot \boldsymbol{x}\left(\boldsymbol{x} \in \Omega_{F}\right)$ of source point. Since $(\boldsymbol{S} \cdot \boldsymbol{x})_{3}=-x_{3}>y_{3}$, the derivation of the Fourier transform $\hat{\overline{\boldsymbol{U}}}_{\infty}$ exploits (16b) and (18b), with $\boldsymbol{x}$ replaced by $\boldsymbol{S} \cdot \boldsymbol{x}$. The value of $\hat{\boldsymbol{U}}_{\infty}$ is then given by (19) with the following modifications: (i) replace $\boldsymbol{q}_{a}^{-}$by $-\boldsymbol{q}_{a}^{+}$, (ii) replace $\boldsymbol{x}$ by $\boldsymbol{S} \cdot \boldsymbol{x}$ and $y_{3}$ by $-y_{3}$, and (iii) right-multiply the result by $\boldsymbol{S}$, to obtain

$$
\hat{\overline{\boldsymbol{U}}}_{\infty}\left(\boldsymbol{\xi}, y_{3}\right)=\hat{\overline{\boldsymbol{U}}}_{\mathrm{P}}(\boldsymbol{\xi}) e^{\boldsymbol{q}_{\mathrm{P}}^{-}(\boldsymbol{\xi}) \cdot \boldsymbol{x}} e^{s_{\mathrm{P}}(\xi) y_{3}}+\hat{\overline{\boldsymbol{U}}}_{\mathrm{S}}(\boldsymbol{\xi}) e^{\boldsymbol{q}_{\mathrm{S}}^{-}(\boldsymbol{\xi}) \cdot \boldsymbol{x}} e^{s_{\mathrm{S}}(\xi) y_{3}}
$$

with

$$
\begin{array}{ll}
\hat{\overline{\boldsymbol{U}}}_{\mathrm{P}}(\boldsymbol{\xi})=\frac{1}{8 \pi^{2} \mu k_{\mathrm{S}}^{2} s_{\mathrm{P}}(\xi)} \boldsymbol{q}_{\mathrm{P}}^{+}(\boldsymbol{\xi}) \otimes \boldsymbol{q}_{\mathrm{P}}^{-}(\boldsymbol{\xi}) & =\boldsymbol{S} \cdot \hat{\boldsymbol{U}}_{\mathrm{P}}(\boldsymbol{\xi}), \\
\hat{\overline{\boldsymbol{U}}}_{\mathrm{S}}(\boldsymbol{\xi})=\frac{-1}{8 \pi^{2} \mu k_{\mathrm{S}}^{2} s_{\mathrm{S}}(\xi)}\left[\boldsymbol{q}_{\mathrm{S}}^{+}(\boldsymbol{\xi}) \otimes \boldsymbol{q}_{\mathrm{S}}^{-}(\boldsymbol{\xi})-k_{\mathrm{S}}^{2} \boldsymbol{S}\right] & =\boldsymbol{S} \cdot \hat{\boldsymbol{U}}_{\mathrm{S}}(\boldsymbol{\xi}),
\end{array}
$$

having used the fact that $\boldsymbol{S} \cdot \boldsymbol{q}_{a}^{+}=-\boldsymbol{q}_{a}^{-}$and $\boldsymbol{q}_{a}^{+} \cdot(\boldsymbol{S} \cdot \boldsymbol{x})=-\boldsymbol{q}_{a}^{-} \cdot \boldsymbol{x}$.

Finally, the traction vector on the free surface associated with $\overline{\boldsymbol{U}}_{\infty}$ is found to be given, in Fourier form, by:

$$
\hat{\overline{\boldsymbol{T}}}_{\infty}(\boldsymbol{\xi})=-\boldsymbol{S} \cdot \hat{\boldsymbol{T}}_{\infty}(\boldsymbol{\xi})
$$

In preparation for formulating the complementary Green's tensor, summing the full-space and image Green's tensors yields

$$
\begin{aligned}
\hat{\boldsymbol{T}}_{\infty}+\hat{\overline{\boldsymbol{T}}}_{\infty}=(\boldsymbol{I}-\boldsymbol{S}) \cdot \hat{\boldsymbol{T}}_{\infty}=2\left(\boldsymbol{e}_{3} \otimes \boldsymbol{e}_{3}\right) & \hat{\boldsymbol{T}}_{\infty} \\
& =\frac{1}{4 \pi^{2} k_{\mathrm{S}}^{2}} \boldsymbol{e}_{3} \otimes\left[2 \boldsymbol{V}_{\mathrm{S}}^{-}(\boldsymbol{\xi}) e^{\boldsymbol{q}_{\mathrm{S}}^{-}(\boldsymbol{\xi}) \cdot \boldsymbol{x}}+\beta(\xi) \boldsymbol{V}_{\mathrm{P}}^{-}(\boldsymbol{\xi}) e^{\boldsymbol{q}_{\mathrm{P}}^{-}(\boldsymbol{\xi}) \cdot \boldsymbol{x}}\right]
\end{aligned}
$$

with $\beta(\xi)=k_{\mathrm{S}}^{2}-2 \xi^{2}=-\left(s_{\mathrm{S}}^{2}(\xi)+\xi^{2}\right)$ and

$$
\boldsymbol{V}_{\mathrm{S}}^{-}(\boldsymbol{\xi}):=-s_{\mathrm{S}}(\xi) \boldsymbol{q}_{\mathrm{S}}^{-}(\boldsymbol{\xi})-k_{\mathrm{S}}^{2} \boldsymbol{e}_{3}, \quad \boldsymbol{V}_{\mathrm{P}}^{-}(\boldsymbol{\xi}):=-s_{\mathrm{P}}(\xi)^{-1} \boldsymbol{q}_{\mathrm{P}}^{-}(\boldsymbol{\xi})
$$

Complementary Green's tensor. Finally, the complementary Green's tensor solves the homogeneous Navier equation in $\Omega_{F}$. Its partial Fourier transform $\hat{\boldsymbol{U}}_{\mathrm{C}}\left(\boldsymbol{\xi}, y_{3}\right)$ thus satisfies

$$
\boldsymbol{A} \hat{\boldsymbol{U}}_{\mathrm{C}}+\boldsymbol{B} \hat{\boldsymbol{U}}_{\mathrm{C}}^{\prime}+\boldsymbol{C} \hat{\boldsymbol{U}}_{\mathrm{C}}^{\prime \prime}=0, \quad\left(y_{3}<0\right),
$$

where the matrix $\boldsymbol{C}$ is defined as in (20) and matrices $\boldsymbol{A}, \boldsymbol{B}$ are given (in tensor form) by

$$
\boldsymbol{A}=\mu\left[\left(1-\kappa^{-2}\right) \boldsymbol{\xi} \otimes \boldsymbol{\xi}-s_{\mathrm{S}}(\xi)^{2} \boldsymbol{I}\right], \quad \boldsymbol{B}=\mathrm{i} \mu\left(\kappa^{-2}-1\right)\left[\boldsymbol{e}_{3} \otimes \boldsymbol{\xi}+\boldsymbol{\xi} \otimes \boldsymbol{e}_{3}\right] .
$$


Moreover, $\hat{\boldsymbol{U}}_{\mathrm{C}}$ must vanish in the limit $y_{3} \rightarrow-\infty$. On enforcing next that such $\hat{\boldsymbol{U}}_{\mathrm{C}}$ actually solves (25), one obtains

$$
\hat{\boldsymbol{U}}_{\mathrm{C}}\left(\boldsymbol{\xi}, y_{3}\right)=\boldsymbol{Q}\left(\boldsymbol{\xi}, y_{3}\right) \cdot \boldsymbol{U}_{\mathrm{C}}^{\mathrm{SP}}
$$

where

$$
\boldsymbol{Q}\left(\boldsymbol{\xi}, y_{3}\right)=\left[\begin{array}{ccc}
\mathrm{i} e^{s_{\mathrm{S}}(\xi) y_{3}} & 0 & \mathrm{i} \xi_{1} e^{s_{\mathrm{P}}(\xi) y_{3}} / s_{\mathrm{P}}(\xi) \\
0 & \mathrm{i} e^{s_{\mathrm{S}}(\xi) y_{3}} & \mathrm{i} \xi_{2} e^{s_{\mathrm{P}}(\xi) y_{3}} / s_{\mathrm{P}}(\xi) \\
\xi_{1} e^{s_{\mathrm{S}}(\xi) y_{3}} / s_{\mathrm{S}}(\xi) & \xi_{2} e^{s_{\mathrm{S}}(\xi) y_{3}} / s_{\mathrm{S}}(\xi) & e^{s_{\mathrm{P}}(\xi) y_{3}}
\end{array}\right]
$$

and the $3 \times 3$ constant matrix $\boldsymbol{U}_{\mathrm{C}}^{\mathrm{SP}}$ is determined by enforcing the free-surface condition

$$
\hat{\boldsymbol{T}}_{\infty}+\hat{\overline{\boldsymbol{T}}}_{\infty}+\hat{\boldsymbol{T}}_{\mathrm{C}}=\mathbf{0} \quad\left(y_{3}=0\right) .
$$

As a result, the complementary Green's tensor is found after a straightforward derivation to be given, in Fourier form, by

$$
\begin{aligned}
\hat{\boldsymbol{U}}_{\mathrm{C}}\left(\boldsymbol{\xi}, y_{3}\right)=\frac{s_{\mathrm{P}}(\xi)}{4 \pi^{2} \delta(\xi) \mu k_{\mathrm{S}}^{2}}\left[2 \boldsymbol{V}_{\mathrm{S}}^{+}(\boldsymbol{\xi}) e^{s_{\mathrm{S}}(\xi) y_{3}}+\beta(\xi) \boldsymbol{V}_{\mathrm{P}}^{+}(\boldsymbol{\xi}) e^{s_{\mathrm{P}}(\xi) y_{3}}\right] & \\
& \otimes\left[2 \boldsymbol{V}_{\mathrm{S}}^{-}(\boldsymbol{\xi}) e^{\boldsymbol{q}_{\mathrm{S}}^{-}(\boldsymbol{\xi}) \cdot \boldsymbol{x}}+\beta(\xi) \boldsymbol{V}_{\mathrm{P}}^{-}(\boldsymbol{\xi}) e^{\boldsymbol{q}_{\mathrm{P}}^{-}(\boldsymbol{\xi}) \cdot \boldsymbol{x}}\right]
\end{aligned}
$$

with $\beta(\xi)$ defined as in $(23), \delta(\xi)$ given by $\delta(\xi):=\beta^{2}(\xi)-4 \xi^{2} s_{\mathrm{P}}(\xi) s_{\mathrm{S}}(\xi)$, and

$$
\boldsymbol{V}_{\mathrm{S}}^{+}(\boldsymbol{\xi})=s_{\mathrm{S}}(\xi) \boldsymbol{q}_{\mathrm{S}}^{+}(\boldsymbol{\xi})+k_{\mathrm{S}}^{2} \boldsymbol{e}_{3}, \quad \boldsymbol{V}_{\mathrm{P}}^{+}(\boldsymbol{\xi})=s_{\mathrm{P}}^{-1}(\xi) \boldsymbol{q}_{\mathrm{P}}^{+}(\boldsymbol{\xi}) .
$$

The unique (real, positive) value $k_{\mathrm{R}}$ of $\xi$ such that $\delta\left(k_{\mathrm{R}}\right)=0$ is the Rayleigh wavenumber (i.e. $k_{\mathrm{R}}$ is, as expected, a pole of $\hat{\boldsymbol{U}}_{\mathrm{C}}$ ), and that one has $k_{\mathrm{P}}<k_{\mathrm{S}}<k_{\mathrm{R}}$. Another important remark is that (26) has an exponential decay of type $O\left(e^{-\xi\left|x_{3}+y_{3}\right|}\right)$ for large $\xi$ provided $x_{3}+y_{3}<0$; this stems from the fact that $s_{a}^{ \pm}(\xi, \alpha)=\xi(1+o(1))$ for sufficiently large $\xi$.

\subsection{Expression in physical coordinates}

The complementary Green's tensor can now be expressed in terms of physical coordinates by means of an inverse Fourier integral:

$$
\boldsymbol{U}_{\mathrm{C}}(\boldsymbol{x}, \boldsymbol{y})=\int_{\mathbb{R}^{2}} e^{\mathrm{i}\left(\xi_{1} y_{1}+\xi_{2} y_{2}\right)} \hat{\boldsymbol{U}}_{\mathrm{C}}\left(\boldsymbol{\xi}, y_{3} ; \boldsymbol{x}\right) \mathrm{d} \xi_{1} \mathrm{~d} \xi_{2} .
$$

Inserting (26) and rearranging terms, the above formula can be recast in the following form:

$$
\boldsymbol{U}_{\mathrm{C}}(\boldsymbol{x}, \boldsymbol{y})=\frac{1}{4 \pi^{2} k_{\mathrm{S}}^{2} \mu} \sum_{a, b=\mathrm{P}, \mathrm{S}} \int_{\mathbb{R}^{2}} A_{a b}(\xi)\left[\exp \left(\boldsymbol{q}_{a}^{+}(\boldsymbol{\xi}) \cdot \boldsymbol{y}\right) \boldsymbol{V}_{a}^{+}(\boldsymbol{\xi})\right] \otimes\left[\exp \left(\boldsymbol{q}_{b}^{-}(\boldsymbol{\xi}) \cdot \boldsymbol{x}\right) \boldsymbol{V}_{b}^{-}(\boldsymbol{\xi})\right] \mathrm{d} \boldsymbol{\xi},
$$

with

$$
A_{\mathrm{PP}}(\xi):=\frac{\beta^{2}(\xi) s_{\mathrm{P}}(\xi)}{\delta(\xi)}, \quad A_{\mathrm{PS}}(\xi)=A_{\mathrm{SP}}(\xi):=\frac{2 \beta(\xi) s_{\mathrm{P}}(\xi)}{\delta(\xi)}, \quad A_{\mathrm{SS}}(\xi):=\frac{4 s_{\mathrm{P}}(\xi)}{\delta(\xi)} .
$$

Decomposition (28) takes the form of a superposition of products of functions of $\boldsymbol{x}$ and of $\boldsymbol{y}$. This structure, reminiscent of the more usual diagonal form available for $\boldsymbol{U}_{\infty}$ [33], is an essential feature for the fast evaluation of (7) and other similar quantities. Numerical evaluation of the integral (28) now requires the definition of an efficient numerical quadrature in Fourier space, which plays the same role as the integration over the unit sphere involved in diagonal-form multipole expansions of free-space fundamental solutions. This task will be facilitated by introducing polar coordinates in the Fourier space, i.e. setting $\boldsymbol{\xi}=\left(\xi_{1}, \xi_{2}\right)=\xi(\cos \alpha, \sin \alpha)$, to obtain

$$
\begin{array}{r}
\boldsymbol{U}_{\mathrm{C}}(\boldsymbol{x}, \boldsymbol{y})=\frac{1}{4 \pi^{2} k_{\mathrm{S}}^{2} \mu} \sum_{a, b=\mathrm{P}, \mathrm{S}} \int_{0}^{+\infty} \xi A_{a b}(\xi)\left\{\int_{0}^{2 \pi}\left[\exp \left(\boldsymbol{q}_{a}^{+}(\xi, \alpha) \cdot \boldsymbol{y}\right) \boldsymbol{V}_{a}^{+}(\xi, \alpha)\right]\right. \\
\left.\otimes\left[\exp \left(\boldsymbol{q}_{b}^{-}(\xi, \alpha) \cdot \boldsymbol{x}\right) \boldsymbol{V}_{b}^{-}(\xi, \alpha)\right] \mathrm{d} \alpha\right\} \mathrm{d} \xi .
\end{array}
$$




\subsection{Discussion}

Partial Fourier vs. Bessel representations of $\boldsymbol{U}_{\boldsymbol{C}}^{\boldsymbol{H} S}$. The angular integrals in (29) can be expressed analytically using formulas (47) involving Bessel functions, reducing (29) to a onedimensional radial integral. Such representation, used in most existing applications of $\boldsymbol{U}^{\mathrm{HS}}$, e.g. [21,28], is however not suitable for a fast-multipole treatment as it destroys the sum-of-products structure of (29). The two-dimensional integral format of (29) is thus an essential feature of the proposed acceleration approach.

A useful consequence of having used the additive decomposition (8) is the fact that $\hat{\boldsymbol{U}}_{\mathrm{C}}\left(\boldsymbol{\xi}, y_{3}\right)$, as given by (26), is a rank-one tensor. This significantly reduces the number of multipole moments that arise from inserting decomposition (29) into (7). Another possible approach, somewhat less attractive from a computational standpoint but nevertheless valid, would consist in directly setting up a partial Fourier representation of $\boldsymbol{U}^{\mathrm{HS}}$ by substituting identities (47) into the Bessel-transform representation of $\boldsymbol{U}^{\mathrm{HS}}$ available e.g. in the appendix of [21], leading to a rank-three tensor function $\hat{\boldsymbol{U}}\left(\boldsymbol{\xi}, y_{3}\right)$.

Convergence of radial integral. The radial integral in (29) is convergent whenever $x_{3}+y_{3}<0$ since (as already mentioned in Sec. 3.1) the integrand in that case decays exponentially as $\xi \rightarrow+\infty$. The case $x_{3}+y_{3}=0$ requires closer examination. To this aim, one notes that $\boldsymbol{V}_{\mathrm{S}}^{ \pm}=O\left(\xi^{2}\right)$, $\boldsymbol{V}_{\mathrm{P}}^{ \pm}=O(1), \beta(\xi)=O\left(\xi^{2}\right)$ and $\delta(\xi)=O\left(\xi^{2}\right)$ (the latter estimate requiring some straightforward derivations) for large $\xi$. The integrand of $(29)$ is thus $O\left(\xi^{5}\right)$ if $x_{3}+y_{3}=0$, making the radial integral divergent. An alternative possibility consists in expressing the complete Green's tensor $\boldsymbol{U}^{\mathrm{HS}}$ in Fourier integral form, with the transform $\hat{\boldsymbol{U}}^{\mathrm{HS}}$ obtained by summing contributions (19), (22) and (26) according to (8). After some manipulation, one finds that $\left\|\hat{\boldsymbol{U}}^{\mathrm{HS}}\left(\xi, \alpha, y_{3}\right)\right\|=O\left(\xi^{-1}\right)$, which still makes the radial integral divergent. The two-dimensional Fourier integral form of $\boldsymbol{U}^{\mathrm{HS}}$, and thus the proposed acceleration approach, is thus valid subject to the restriction $x_{3}+y_{3}<0$ and cannot be applied when $x_{3}+y_{3}=0$. Furthermore, if the angular integrations are effected analytically first (using formulae (47)), the integrands in the resulting radial integrals can be shown (from the large-argument asymptotic behavior of the Bessel functions, see e.g. formulae 8.451 in [17]) to be equivalent to a linear combination of $(2 / \xi r)^{1 / 2} \cos \xi r$ and $(2 / \xi r)^{1 / 2} \sin \xi r$ (with $\left.r:=\|\boldsymbol{y}-\boldsymbol{x}\|\right)$ ). The corresponding radial integrals are known to be finite (see e.g. formulae 3.751 in [17]). As a result, the non-multipole evaluation of $\boldsymbol{U}^{\mathrm{HS}}$ by means of radial Fourier integration remains available in this case. One may thus conclude that configuration involving pairs of source and observation points that are both located on the surface $\Gamma_{F}$ are permitted, but any Green's tensor evaluations involving such pairs must be effected in non-multipole fashion.

Clustering. If convergent (i.e. whenever $x_{3}+y_{3}<0$ ), integral (29) can be evaluated accurately by means of a suitably-constructed quadrature rule for any configuration of source and observation points. In particular, in contrast with more usual forms of the FMM, integral representation (29) of $\boldsymbol{U}_{\mathrm{C}}$ is valid without requiring that clusters of source and observation points be well-separated (both clusters may well be in fact identical). As a consequence, there is no obligation to define subsets of points through a division of the spatial region enclosing all points into cubic cells. The simplest option of applying (29) to all source and observation points at once (i.e., in FMM parlance, of enclosing all points in one single cell) is available, and is adopted for the remainder of this article.

\section{Quadrature strategy for fast evaluation of elastodynamic potentials}

\subsection{Fast Multipole Method}

The evaluation of a single-layer potential $\mathcal{S}[\boldsymbol{t}](\boldsymbol{x})$ of the form (7) for a given density $\boldsymbol{t}$, which is typically one of the main computational tasks involved in the iterative solution of integral equations such as (6), is now addressed. As indicated earlier, attention is focused on the computation of the complementary potential $\mathcal{S}_{\mathrm{C}}[\boldsymbol{t}](\boldsymbol{x})$ introduced in (10), as the other contributions $\mathcal{S}_{\infty}[\boldsymbol{t}](\boldsymbol{x})$ and 
$\overline{\mathcal{S}}_{\infty}[\boldsymbol{t}](\boldsymbol{x})$ can be evaluated using known FMM procedures. Substituting the representation (29) of $\boldsymbol{U}_{\mathrm{C}}$ into the definition of $\mathcal{S}_{\mathrm{C}}[\boldsymbol{t}](\boldsymbol{x})$ and rearranging terms, the complementary potential is given by

$$
\mathcal{S}_{\mathrm{C}}[\boldsymbol{t}](\boldsymbol{x})=\frac{1}{4 \pi^{2} k_{\mathrm{S}}^{2} \mu} \sum_{a, b=\mathrm{P}, \mathrm{S}} \int_{0}^{+\infty} \xi A_{a b}(\xi)\left\{\int_{0}^{2 \pi} \mathcal{R}_{a}(\xi, \alpha)\left[\exp \left(\boldsymbol{q}_{b}^{-}(\xi, \alpha) \cdot \boldsymbol{x}\right) \boldsymbol{V}_{b}^{-}(\xi, \alpha)\right] \mathrm{d} \alpha\right\} \mathrm{d} \xi
$$

where the multipole moments are given by

$$
\mathcal{R}_{a}(\xi, \alpha):=\left\{\int_{S} \exp \left(\boldsymbol{q}_{a}^{+}(\xi, \alpha) \cdot \boldsymbol{y}\right) \boldsymbol{t}(\boldsymbol{y}) \mathrm{d} S_{y}\right\} \cdot \boldsymbol{V}_{a}^{+}(\xi, \alpha) .
$$

The Fourier integral (30) is in practice evaluated by means of a product quadrature rule, whose details will be discussed next, so that one has

$$
\mathcal{S}_{\mathrm{C}}[\boldsymbol{t}](\boldsymbol{x})=\frac{1}{4 \pi^{2} k_{\mathrm{S}}^{2} \mu} \sum_{a, b=\mathrm{P}, \mathrm{S}} \sum_{i=1}^{n_{\xi}} w_{i}^{\xi} \xi_{i} A_{a b}\left(\xi_{i}\right)\left\{\sum_{j=1}^{n_{\alpha}} w_{j}^{\alpha} \mathcal{R}_{a}\left(\xi_{i}, \alpha_{j}\right)\left[\exp \left(\boldsymbol{q}_{b}^{-}\left(\xi_{i}, \alpha_{j}\right) \cdot \boldsymbol{x}\right) \boldsymbol{V}_{b}^{-}\left(\xi_{i}, \alpha_{j}\right)\right]\right\}
$$

where $\left(\xi_{i}, w_{i}^{\xi}\right)_{1 \leq i \leq n_{\xi}}$ and $\left(\alpha_{j}, w_{j}^{\alpha}\right)_{1 \leq j \leq n_{\alpha}}$ denote the sets of nodes and weights used for the radial and angular quadratures, respectively, and $E\left(n_{\xi}, n_{\alpha}\right)$ is the quadrature error.

The computational complexity of the evaluation of $\mathcal{S}_{\mathrm{C}}[\boldsymbol{t}](\boldsymbol{x})$ by means of (32) is of order $O\left(n_{\xi} n_{\alpha} N\right)$ (where $n_{\xi}$ and $n_{\alpha}$ depend on $N$, as discussed in Sec. 6), since the computational work required by (31) and (32) is (for a fixed quadrature rule) clearly proportional to the $O(N)$ size of the set of collocation points and that of the BE mesh, respectively. In contrast, when evaluating $\mathcal{S}_{\mathrm{C}}[\boldsymbol{t}](\boldsymbol{x})$ in standard (non-multipole) fashion, no angular quadrature is required thanks to the exact formulae (47) but $\boldsymbol{x}$ and $\boldsymbol{y}$ are no longer separated in $\boldsymbol{U}_{\mathrm{C}}(\boldsymbol{x}, \boldsymbol{y})$, resulting in an overall computational complexity of order $O\left(n_{\xi} N^{2}\right)$.

\subsection{Behavior of the integrands in the multipole expansion}

The construction of quadrature rules suited to the evaluation of (32) is dictated by the following considerations.

Radial integration: truncation. As already mentioned in Sec. 3.1, the large- $\xi$ behavior of $\hat{\boldsymbol{U}}^{\mathrm{HS}}\left(\xi, \alpha, y_{3}\right)$ is of type $O\left(e^{-\xi\left|x_{3}+y_{3}\right|}\right)$ provided $x_{3}+y_{3}<0$. The radial integral in (29) may thus be truncated to a finite interval $\xi \in\left[0, \xi_{\max }\right]$. The upper bound $\xi_{\max }$ is here set to the following value, similar to the one used in [28]:

$$
\xi_{\max }=2 k_{\mathrm{R}}+\frac{10}{\min \left|x_{3}\right|} \quad\left(k_{\mathrm{R}} \text { is the Rayleigh wavenumber }\right) .
$$

Radial integration: Rayleigh pole. The radial integral in (29) has one branch point at $\xi=k_{\mathrm{P}}$ and one pole at $\xi=k_{\mathrm{R}}$. The Rayleigh pole, which corresponds to the velocity of Rayleigh waves propagating along the free surface, may cause difficulties in the quadrature as it lies on the real $\xi$-axis. Its contribution to the radial integral may be evaluated by means of the residue theorem (see e.g. [13]) or by using the analyticity of the integrand and deforming the integration path to avoid the singularity (see [22], where this treatment is used for layered viscoelastic media). A simpler approach, which consists in considering the elastic material as the limiting case of a viscoelastic material with vanishingly small attenuation [28], is used here through setting the angular frequency to the complex value $\omega(1+i \gamma)$ with $\gamma=10^{-8}$. The Rayleigh singularity on the real $\xi$-axis is thus avoided.

Radial integration: oscillatory integrands. The radial integrands in (29) are also oscillatory. Classical (e.g. Gaussian) quadrature rules over the whole interval $\xi \in\left[0, \xi_{\max }\right]$ perform poorly for highly oscillatory integrands. This difficulty may be overcome by splitting $\xi \in\left[0, \xi_{\max }\right]$ into many subintervals; the resulting computational burden however becomes unacceptable in the context of 
the FMM. Alternatively, quadrature rules whose weights incorporate analytically-computed contributions of the oscillatory factor in the integrand, initially proposed in [15], may be used (see e.g. [28] for the non-multipole computation of $\boldsymbol{e}_{3} \cdot \boldsymbol{U}^{\mathrm{HS}}$ ). This approach is however not suitable for a FMM framework since (for the present context) it relies on Bessel transforms and thus destroys the separation of variables in (29).

Angular integration. Integrals over $\alpha$ involved in (29) are of the form

$$
I_{n, m}:=\int_{0}^{2 \pi} e^{\mathrm{i} \xi\left(r_{1} \cos \alpha+r_{2} \sin \alpha\right)} \cos ^{n} \alpha \sin ^{m} \alpha \mathrm{d} \alpha \quad(0 \leq n, m \leq 2,0 \leq n+m \leq 2) .
$$

They involve smooth periodic functions. Trapezoidal quadrature rules are well suited to this type of integral, and are used here; they were also used in [7] for radial Fourier integrals involving $\hat{G}$ given by $(16 a, b)$.

\subsection{Generalized Gaussian approach for radial quadrature}

The previous considerations highlight the need for quadrature rules that perform adequately and efficiently for the radial integrals, which are both oscillatory and singular. The framework of generalized Gaussian quadrature [4,8,36] (GGQ) allows to generate rules that are custom-tailored to specific types of problematic integrands such as those involved here, and require much fewer nodes than standard rules. The Fourier representation (29) of $\boldsymbol{U}_{\mathrm{C}}$ involves a singularity not present in corresponding representations for Helmholtz problems, namely the Rayleigh pole $\xi=k_{\mathrm{R}}$. As a result, the tabulated GGQ rules given in $[4,27,36]$ cannot be used directly here. Instead, the methodology for GGQ generation described in [4] is specialized to, and implemented for, the class of integrands involved in the evaluation of (29), as described next in Sec. 5. The version of [4] is preferred here as their authors deem it to be more robust than their earlier versions $[27,36]$ regarding the treatment of singularities appearing in (29).

Principle of generalized Gaussian quadratures. The radial integration featured in (29) requires a quadrature rule that is suitable for a set of $L$ specific integrals of the form

$$
I_{\ell}(\boldsymbol{p})=\int_{0}^{\xi \max } f_{\ell}(\xi ; \boldsymbol{p}) \mathrm{d} \xi \quad(1 \leq \ell \leq L, \boldsymbol{p} \in \mathcal{P})
$$

where $\left(f_{\ell}\right)_{1 \leq \ell \leq L}$ are real-valued square-integrable functions, whose (singular, oscillatory...) behavior may make numerical quadrature difficult (or a priori expensive), and $\boldsymbol{p}$ is a set of parameters that enter the functions. The $f_{\ell}$ are chosen so that all integrands appearing in (29) are linear combinations of them (the $L=17$ such functions needed for this purpose are listed in Appendix C), while the parameters gathered in $\boldsymbol{p}$ are the frequency $\omega$ and the source and observation points $\boldsymbol{x}, \boldsymbol{y}$. The quadrature rule then must yield accurate values of the integrals $I_{\ell}(\boldsymbol{p})$ for all values of $\boldsymbol{p}$ in a given parameter domain $\mathcal{P}$, whose definition reflects the clusters of source and observation points for which $\boldsymbol{U}_{\mathrm{C}}(\boldsymbol{x}, \boldsymbol{y})$ needs to be evaluated and the frequencies of interest. To this aim, a large number $K$ of sampling values $\boldsymbol{p}_{k} \in \mathcal{P}$ is introduced, so as to define a large collection of $m=K L$ input functions $\phi_{i}(\xi)(1 \leq i \leq m)$, each $\phi_{i}$ being of the form $\phi_{i}(\xi)=f_{\ell}\left(\xi ; \boldsymbol{p}_{k}\right)$ for some $(k, \ell)$. GGQ rules are then constructed so as to integrate accurately the whole collection of input functions $\phi_{1}, \ldots, \phi_{m}$. For a sufficiently dense sampling of $\mathcal{P}$, the same level of accuracy will then be achieved on $\boldsymbol{U}_{\mathrm{C}}(\boldsymbol{x}, \boldsymbol{y})$ for any $(\boldsymbol{x}, \boldsymbol{y})$ within the prescribed parameter range.

Parameters. To specify the parameters involved in (29) and their domain $\mathcal{P}$, it is convenient to use non-dimensional quantities. Accordingly, setting $\xi=k_{\mathrm{S}} \bar{\xi}$ (where $\bar{\xi}$ is non-dimensional), the other quantities entering (29) can be expressed in terms of non-dimensional counterparts as follows: $\xi \mathrm{d} \xi=k_{\mathrm{S}}^{2} \bar{\xi} \mathrm{d} \bar{\xi}, s_{a}=k_{\mathrm{S}} \bar{s}_{a}(\bar{\xi}), \boldsymbol{q}_{a}^{ \pm}(\xi, \alpha)=k_{\mathrm{S}} \overline{\boldsymbol{q}}_{a}^{ \pm}(\bar{\xi}, \alpha), \boldsymbol{V}_{\mathrm{P}}^{ \pm}(\xi, \alpha)=\overline{\boldsymbol{V}}_{\mathrm{P}}^{ \pm}(\bar{\xi}, \alpha), \boldsymbol{V}_{\mathrm{S}}^{ \pm}(\xi, \alpha)=k_{\mathrm{S}}^{2} \overline{\boldsymbol{V}}_{\mathrm{S}}^{ \pm}(\bar{\xi}, \alpha)$, 
$A_{\mathrm{PP}}(\xi)=k_{\mathrm{S}}^{2} \bar{A}_{\mathrm{PP}}(\bar{\xi}), A_{\mathrm{PS}}(\xi)=\bar{A}_{\mathrm{PS}}(\bar{\xi}), A_{\mathrm{SS}}(\xi)=k_{\mathrm{S}}^{-2} \bar{A}_{\mathrm{SS}}(\bar{\xi})$, where all overbarred quantities are non-dimensional and depend only on the non-dimensional integration variable $\bar{\xi}$. Expression (29) then takes the form

$$
\begin{array}{r}
\boldsymbol{U}_{\mathrm{C}}(\boldsymbol{x}, \boldsymbol{y})=\frac{k_{\mathrm{S}}^{2}}{4 \pi^{2} \mu} \sum_{a, b=\mathrm{P}, \mathrm{S}} \int_{0}^{+\infty} \bar{\xi} \bar{A}_{a b}(\bar{\xi})\left\{\int_{0}^{2 \pi}\left[\exp \left(k_{\mathrm{S}} \overline{\boldsymbol{q}}_{a}^{+}(\bar{\xi}, \alpha) \cdot \boldsymbol{y}\right) \overline{\boldsymbol{V}}_{a}^{+}(\bar{\xi}, \alpha)\right]\right. \\
\left.\otimes\left[\exp \left(k_{\mathrm{S}} \overline{\boldsymbol{q}}_{b}^{-}(\xi, \alpha) \cdot \boldsymbol{x}\right) \overline{\boldsymbol{V}}_{b}^{-}(\bar{\xi}, \alpha)\right] \mathrm{d} \alpha\right\} \mathrm{d} \bar{\xi} .
\end{array}
$$

Apart from the multiplicative factor $k_{\mathrm{S}}^{2} /\left(4 \pi^{2} \mu\right)$, which affects neither the quadrature definition nor the relative quadrature error, the parameters $\omega, \boldsymbol{x}$ and $\boldsymbol{y}$ appear in the above integrand only in the two exponentials. Since, by virtue of definitions (17), one has

$$
\begin{aligned}
\exp \left(k_{\mathrm{S}} \overline{\boldsymbol{q}}_{a}^{+}(\bar{\xi}, \alpha) \cdot \boldsymbol{y}\right) & \exp \left(k_{\mathrm{S}} \overline{\boldsymbol{q}}_{b}^{-}(\xi, \alpha) \cdot \boldsymbol{x}\right) \\
& =\exp \left(\mathrm{i} k_{\mathrm{S}}\left[\bar{\xi}_{1}\left(y_{1}-x_{1}\right)+\bar{\xi}_{2}\left(y_{2}-x_{2}\right)\right]\right) \exp \left(k_{\mathrm{S}}\left(\bar{s}_{a} y_{3}+\bar{s}_{b} x_{3}\right)\right) \quad(a, b=\mathrm{P}, \mathrm{S}),
\end{aligned}
$$

the relevant non-dimensional parameters involved in (35) are

$$
p_{1}:=k_{\mathrm{S}}\left[\left(y_{1}-x_{1}\right)^{2}+\left(y_{2}-x_{2}\right)^{2}\right]^{1 / 2}, \quad p_{2}:=k_{\mathrm{S}} x_{3}, \quad p_{3}:=k_{\mathrm{S}} y_{3} .
$$

The parameter set $\mathcal{P}$ may then be defined as a set of points in $\left(p_{1}, p_{2}, p_{3}\right)$ space. One notes in particular that dimensional invariance implies that the same parameter set $\mathcal{P}$ corresponds to sets of points $\boldsymbol{x}, \boldsymbol{y}$ in physical space whose size may vary in inverse relation to the chosen frequency. Moreover, if all points $\boldsymbol{x}$ and $\boldsymbol{y}$ of interest are located on the same geometrical entity (e.g. the surface $S$ for potentials (7)), parameters $p_{1}$ and $p_{2}$ are sufficient to specify limits on $\omega, \boldsymbol{x}$ and $\boldsymbol{y}$.

Overview of the GGQ-generating algorithm. The required GGQ rule is generated by the procedure outlined in Box 2. It produces a set of $n_{\xi}$ quadrature nodes and weights $\left(\xi_{j}, w_{j}^{\xi}\right)_{1 \leq j \leq n_{\xi}}$ (with $n_{\xi}$ much smaller than $m$ ) such that

$$
\left|\sum_{j=1}^{n_{\xi}} \phi_{i}\left(\xi_{j}\right) w_{j}^{\xi}-\int_{0}^{\xi_{\max }} \phi_{i}(\xi) \mathrm{d} \xi\right| \leq \varepsilon \quad(1 \leq i \leq m) .
$$

This procedure directly transposes for the present needs the GGQ generation method of [4], to which the reader is referred for theoretical background.

\section{Construction of the custom-tailored generalized Gaussian quadrature}

This section is devoted to a detailed explanation of the four main steps of the GGQ-generating procedure used in this work (Box 2) and their implementation.

The FMM algorithm requires a single quadrature rule for the nine scalar components of $\boldsymbol{U}_{\mathrm{C}}$ as given by (29). The latter can be expressed as linear combinations of a set of 17 functions $f_{1}\left(\xi ; p_{1}, p_{2}\right), \ldots, f_{17}\left(\xi ; p_{1}, p_{2}\right)$, given in Appendix C. The $m=17 K$ input functions $\phi_{1}(\xi), \ldots, \phi_{m}(\xi)$ are then defined from the $f_{\ell}\left(\xi ; p_{1}, p_{2}\right)$ by sampling the parameter region of interest $\mathcal{P}$ using a large number $K$ of values $\left(p_{1}, p_{2}\right)_{k} \in \mathcal{P}, 1 \leq k \leq K$.

Step 1: adaptive construction of an oversized quadrature. A piecewise-Legendre quadrature rule integrating all products of input functions is constructed. Its $p$ nodes $\hat{\xi}_{k}$ and weights $\hat{w}_{k}$ are determined so that these integrals are computed with precision $\varepsilon_{1}$, i.e.

$$
\left|\int_{0}^{\xi \max } \phi_{i}(\xi) \phi_{j}(\xi) \mathrm{d} \xi-\sum_{k=1}^{p} \phi_{i}\left(\hat{\xi}_{k}\right) \phi_{j}\left(\hat{\xi}_{k}\right) \hat{w}_{k}^{\xi}\right| \leq \varepsilon_{1} \quad(1 \leq i, j \leq m) .
$$


Box 2 Generation of generalized Gaussian quadrature rules: main steps of the algorithm.

Input $m$ input functions $\phi_{1}, \ldots, \phi_{m}$ defined on the interval $\left[0, \xi_{\max }\right]$ (Appendix $\mathrm{C}$ )

Step 1 Compute nodes $\hat{\xi}_{1}, \ldots, \hat{\xi}_{p}$ and weights $\hat{w}_{1}, \ldots, \hat{w}_{p}$ of a $p$-node piecewise-Legendre quadrature rule (with $p$ large) that integrates accurately all products of input functions $\left(\phi_{i}\right)_{1 \leq i \leq m}$ (Algorithm 1).

Step 2 Find the (low) numerical rank $r$ of the set of input functions; compress input functions to $r L^{2}$-orthonormal functions $u_{1}, \ldots, u_{r}$ (Algorithm 2).

Step 3 Construct nodes $\tilde{\xi}_{1}, \ldots, \tilde{\xi}_{r}$ and weights $\tilde{w}_{1}, \ldots, \tilde{w}_{r}$ of a $r$-point quadrature rule that achieves the same accuracy as Step 1 (Algorithm 3).

Step 4 Further reduce the number of nodes from $r$ to $n_{\xi}$ using non-quadratic optimization (Algorithm 4).

Output $n_{\xi}$-point GGQ rule (nodes $\xi_{1}, \ldots, \xi_{n_{\xi}}$, weights $w_{1}^{\xi}, \ldots, w_{n_{\xi}}^{\xi}$ )

Since the set $f_{1}, \ldots, f_{17}$ is exhaustive with respect to the radial integration in (29), the $p$-node quadrature will then evaluate accurately $\boldsymbol{U}_{\mathrm{C}}(\boldsymbol{x}, \boldsymbol{y})$ for any $\boldsymbol{x}, \boldsymbol{y}$ covered in $\mathcal{P}$ provided the sampling of $\mathcal{P}$ is fine enough (i.e. $K$ is large enough). The nodes $\hat{\xi}_{k}$ and weights $\hat{w}_{k}$ in (37) are obtained by adaptively constructing a piecewise Legendre quadrature (Algorithm 1). An order- $\ell$ Legendre expansion on $\left[0, \xi_{\max }\right]$ is sought for each $\phi_{i}$. If this expansion is satisfactory (which is decided on the basis of the $L^{2}$-norm of the difference between order- $\ell$ and order- $2 \ell$ being smaller than a preset tolerance, see step 5 of Algorithm 1), the iterative process is stopped; otherwise the interval is split into two subintervals of equal length and order- $\ell$ Legendre expansions are sought on each subinterval until either no further subdivision is found necessary, or the maximum number of successive subdivisions (here set to 30 ) is reached. The order $\ell$, which is an adjustable parameter of the algorithm, must be large enough for detecting singularities yet small enough for the algorithm to be efficient ( $\ell=20$ is used here, while examples in [4] use $\ell=30$ ). Because classical quadratures perform poorly for integrating singular and oscillatory functions, the number $p$ of nodes generated for this preliminary quadrature is quite large (typically of the order of $10^{3}$ ).

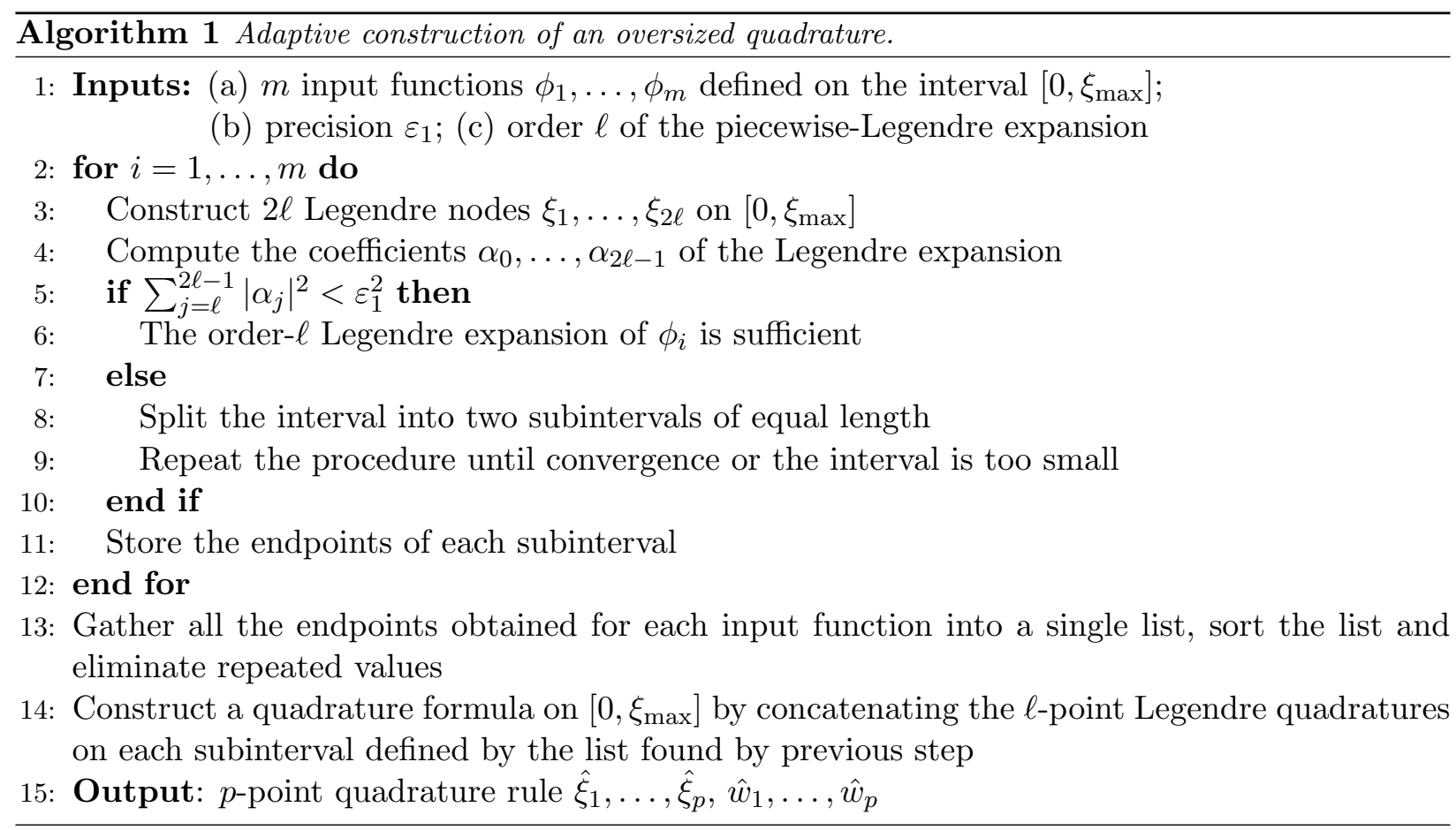




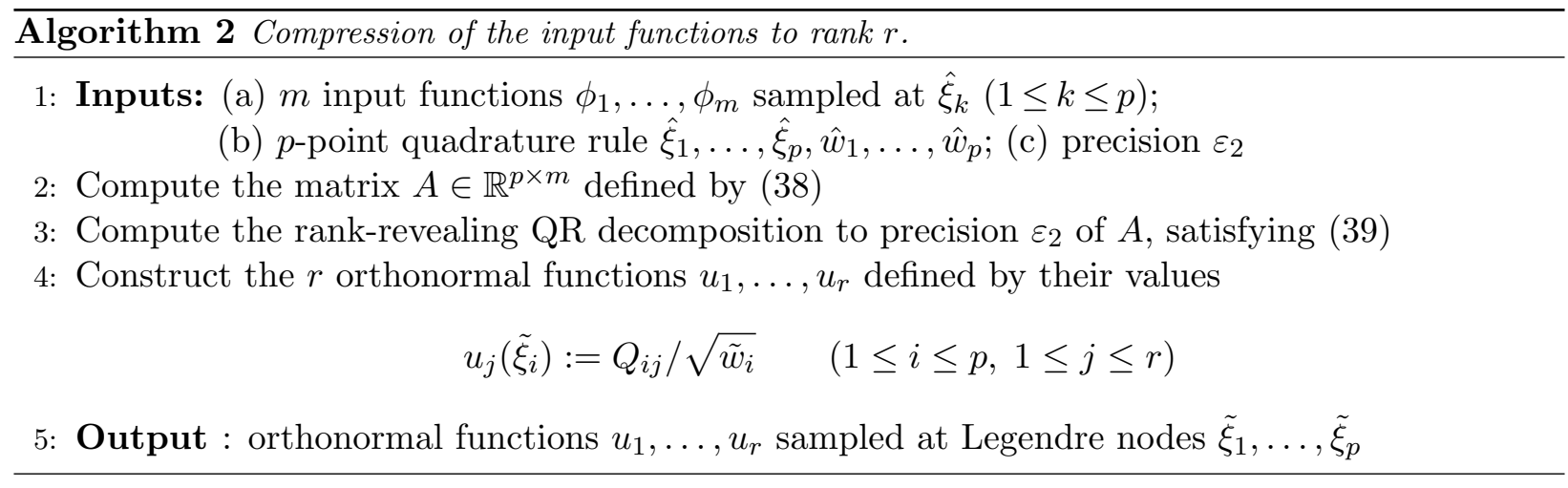

Step 2: compression of the input functions to rank $r$. The unacceptably large size $p$ of the quadrature rule produced by Step 1 is now reduced by observing that the values $\phi_{i}\left(\hat{\xi}_{k}\right)$ of the input functions sampled at the $p$ nodes $\hat{\xi}_{k}$ are highly redundant, i.e. that the matrix $A$, defined by

$$
A \in \mathbb{R}^{p \times m}, \quad A_{k i}:=\phi_{i}\left(\hat{\xi}_{k}\right) \sqrt{\hat{w}_{k}}
$$

has a numerical rank $r$ much smaller than both $p$ and $m$. Accordingly, an orthonormal basis $u_{1}, \ldots, u_{r}$ of functions is built, and $r$ evaluated, by applying a rank-revealing QR decomposition to $A$ (see details in Algorithm 2) with precision $\varepsilon_{2}$, i.e. such that

$$
\|A-Q R\|_{2} \leq \varepsilon_{2} .
$$

The initial large set of input functions $\phi_{1}, \ldots, \phi_{m}$ is thereby compressed into the smaller set $u_{1}, \ldots, u_{r}$ of (sampled) functions.

Step 3: construction of a $r$-point quadrature rule. A $r$-point quadrature rule $\tilde{\xi}_{1}, \ldots \tilde{\xi}_{r}$, $\tilde{w}_{1}, \ldots, \tilde{w}_{r}$ that accurately integrates the $r$ orthonormal functions $u_{1}, \ldots, u_{r}$ can now be extracted from the $p$-point quadrature. The procedure (Algorithm 3) consists in finding the minimum-norm solution $Z \in \mathbb{R}^{p}$ of the underdetermined system of equations

$$
B Z=R,
$$

with the matrix $B \in \mathbb{R}^{r \times p}$ and the vector $R \in \mathbb{R}^{r}$ given by

$$
\begin{aligned}
B_{i k} & :=u_{i}\left(\hat{\xi}_{k}\right) \sqrt{\hat{w}_{k}} & & (1 \leq i \leq r, 1 \leq k \leq p), \\
R_{i} & :=\sum_{k=1}^{p} u_{i}\left(\hat{\xi}_{k}\right) \hat{w}_{k} \approx \int_{0}^{\xi \max } u_{i}(\xi) \mathrm{d} \xi & & (1 \leq i \leq r),
\end{aligned}
$$

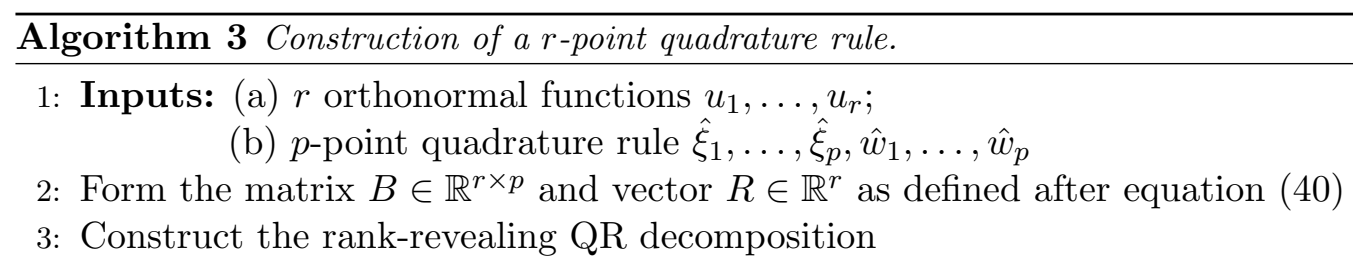

$$
B \Pi=Q\left[R_{11} R_{12}\right]
$$

(with $Q \in \mathbb{R}^{r \times r}$ orthogonal, $R_{11} \in \mathbb{R}^{r \times r}$ upper triangular, and $\Pi \in \mathbb{R}^{p \times p}$ a permutation)

4: Compute the solution $Z \in \mathbb{R}^{r}$ of $R_{\tilde{\xi}_{1}} Z=\widetilde{Q}^{*} r$ using backsubstitution

5: Form the new $r$-point quadrature $\tilde{\xi}_{1}, \ldots, \tilde{\xi}_{r}, \tilde{w}_{1}, \ldots, \tilde{w}_{r}$ by setting

$$
\tilde{\xi}_{k}=\hat{\xi}_{i_{k}} \text { and } \tilde{w}_{k}=z_{k} \hat{w}_{i_{k}}
$$

where $i_{k}$ denotes the row index of the non zero entry in the $k$-th column of $\Pi$

6: Output : $r$-point quadrature rule $\tilde{\xi}_{1}, \ldots, \tilde{\xi}_{r}, \tilde{w}_{1}, \ldots, \tilde{w}_{r}$ 
and defining the nodes and weights on the basis of the $r$ non-zero entries $Z_{i_{1}}, \ldots, Z_{i_{r}}$ of $Z$ by

$$
\tilde{\xi}_{k}=\hat{\xi}_{i_{k}}, \quad \tilde{w}_{k}=Z_{i_{k}} \hat{w}_{i_{k}} .
$$

This $r$-point quadrature rule also accurately integrates the input functions $\phi_{1}, \ldots, \phi_{m}$. The size $r$ of this quadrature rule, equal to the rank of the input functions sampled at the Legendre nodes, was usually observed to be in the 10-100 range, i.e. about 10 to 100 times smaller than $p$.

Step 4: Point-by-point reduction of quadrature using non-quadratic optimization. Observing that the $n_{\xi}$ nodes $\xi_{k}$ and weights $w_{k}$ of a quadrature exact for the input functions $\left(\phi_{i}\right)_{1 \leq i \leq m}$ satisfy the nonlinear system of equations,

$$
R\left(\xi_{1}, \ldots, \xi_{n}, w_{1}, \ldots, w_{n} ; \phi_{i}\right):=\sum_{k=1}^{n_{\xi}} \phi_{i}\left(\xi_{k}\right) w_{k}-\int_{0}^{\xi \max } \phi_{i}(\xi) \mathrm{d} \xi=0 \quad(1 \leq i \leq m),
$$

the reduction of the number of quadrature nodes is a non-quadratic optimization problem whereby equations (41) applied to $u_{1}, \ldots u_{r}$ instead of $\phi_{1}, \ldots, \phi_{m}$ are to be satisfied in the least-squares sense. Beginning with the quadrature rule $\tilde{\xi}_{1}, \ldots, \tilde{\xi}_{r}, \tilde{w}_{1}, \ldots, \tilde{w}_{r}$ produced by Algorithm 3 , a procedure reducing a $n$-point quadrature rule $\xi_{1}, \ldots, \xi_{n}, w_{1}, \ldots, w_{n}$ to a $(n-1)$-point quadrature rule by removing one node and rearranging the others so as to satisfy (41) in the least-squares sense is repeatedly applied until (41) can no longer be satisfied within a preset precision $\varepsilon_{3}$. Algorithm 4 implements this procedure in two steps.

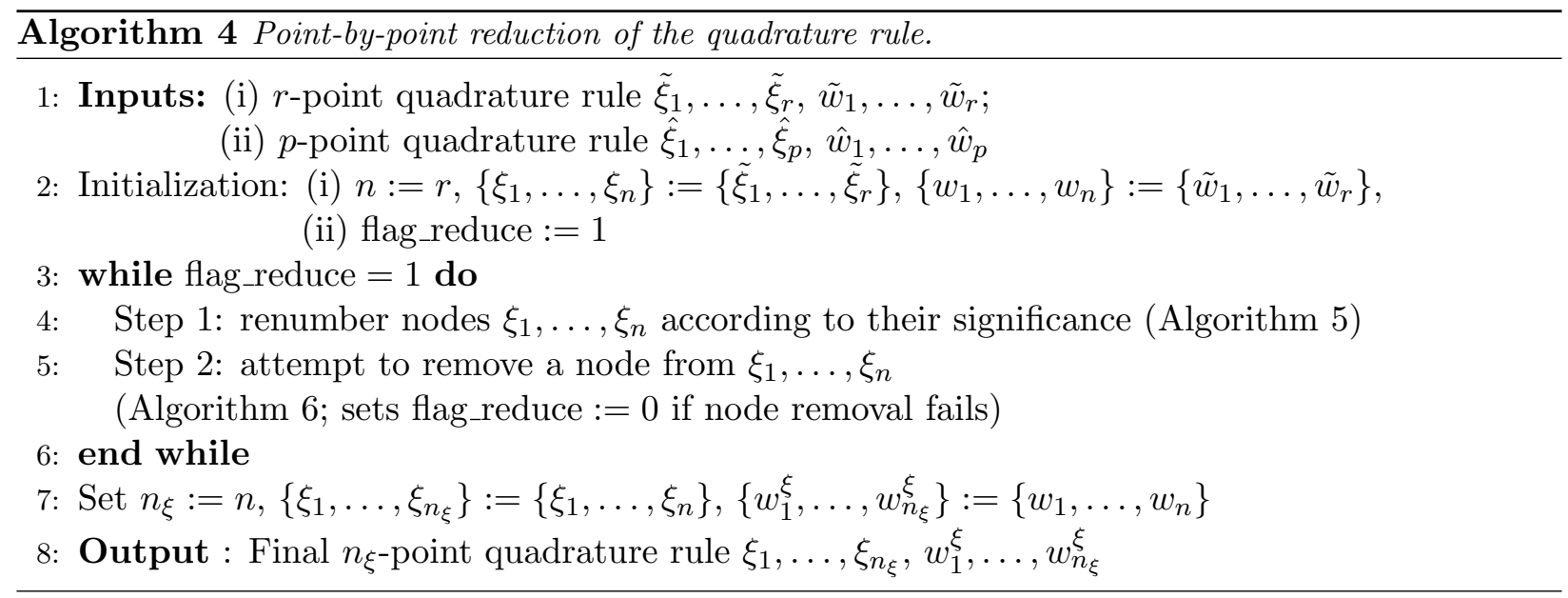

In step 1 of Algorithm 4, the significance $\eta_{j}$ of a quadrature node $\xi_{j}$ of the current $n$-point rule is defined, for given $j$, as $\eta_{j}:=\left\|\Delta z^{(j)}\right\|$, where $\Delta z^{(j)}$ is the Gauss-Newton step direction associated with minimizing the $L^{2}$-norm of the $r$-vector of residuals $\left\{R\left(\left\{\xi_{1}, \ldots, \xi_{n}\right\} \backslash \xi_{j},\left\{w_{2}, \ldots, w_{n}\right\} \backslash w_{j} ; u_{i}\right)\right\}$ after removing $\left(\xi_{j}, w_{j}\right)$ from the current rule. The significance $\eta_{j}$ thus measures the perturbation induced to the quadrature rule by removal of node $\xi_{j}$. In practice, $\Delta z^{(j)}$ is found as the minimumnorm solution of the underdertermined linear least-squares problem ${ }^{1} \min _{z}\left\|J^{(j)} z-\Delta R^{(j)}\right\|^{2}$ (with notations as in Algorithm 5). Algorithm 5 implements step 1, i.e. computes all $\eta_{j}$ and reorders $\xi_{1}, \ldots, \xi_{n}$ and $w_{1}, \ldots, w_{n}$ along increasing $\eta_{j}$.

Then (step 2 of Algorithm 4), to actually downsample the current $n$-point quadrature rule by one node, the least-significant node $\xi_{1}$ is removed from the quadrature rule and equations (41) are solved in the least-squares sense (using Gauss-Newton iterations) for $\xi_{2}, \ldots, \xi_{n}, w_{2}, \ldots, w_{n}$, in the expectation that removing the least-significant node maximises the chances of obtaining a $n$-1-node quadrature that achieves an acceptably small norm for the vector of residuals

\footnotetext{
${ }^{1}$ There seems to be an error in [4] where the authors state $\Delta z^{(j)}=\operatorname{argmin}_{z}\left\|J^{(j)} z-R\right\|_{2}$
} 


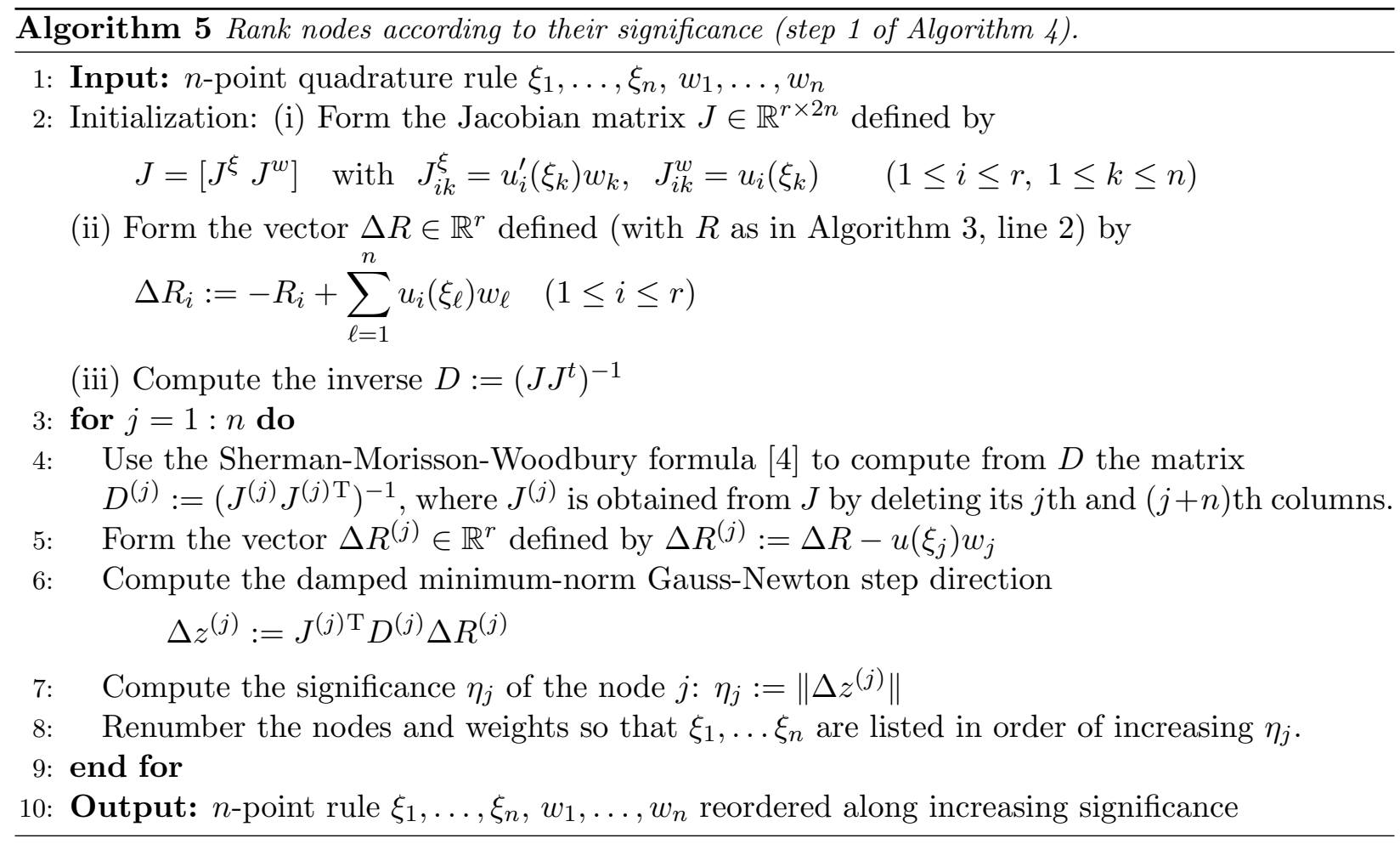

$R\left(\xi_{2}, \ldots, \xi_{n}, w_{2}, \ldots, w_{n} ; u_{i}\right)(1 \leq i \leq r)$. If the removal of $\xi_{1}$ proves unsussessful, it is added back to the quadrature, and $\xi_{2}$ is removed instead, and so on until either one node removal is successful (this removal is then made permanent, and the new $n-1$ quadrature is used as starting point for the next downsampling attempt) or all node removals are unsuccessful (the quadrature before removal is then deemed to be the final GGQ rule). The iterative node removal producing the final GGQ rule (i.e. step 2 of Algorithm 4) is implemented in Algorithm 6.

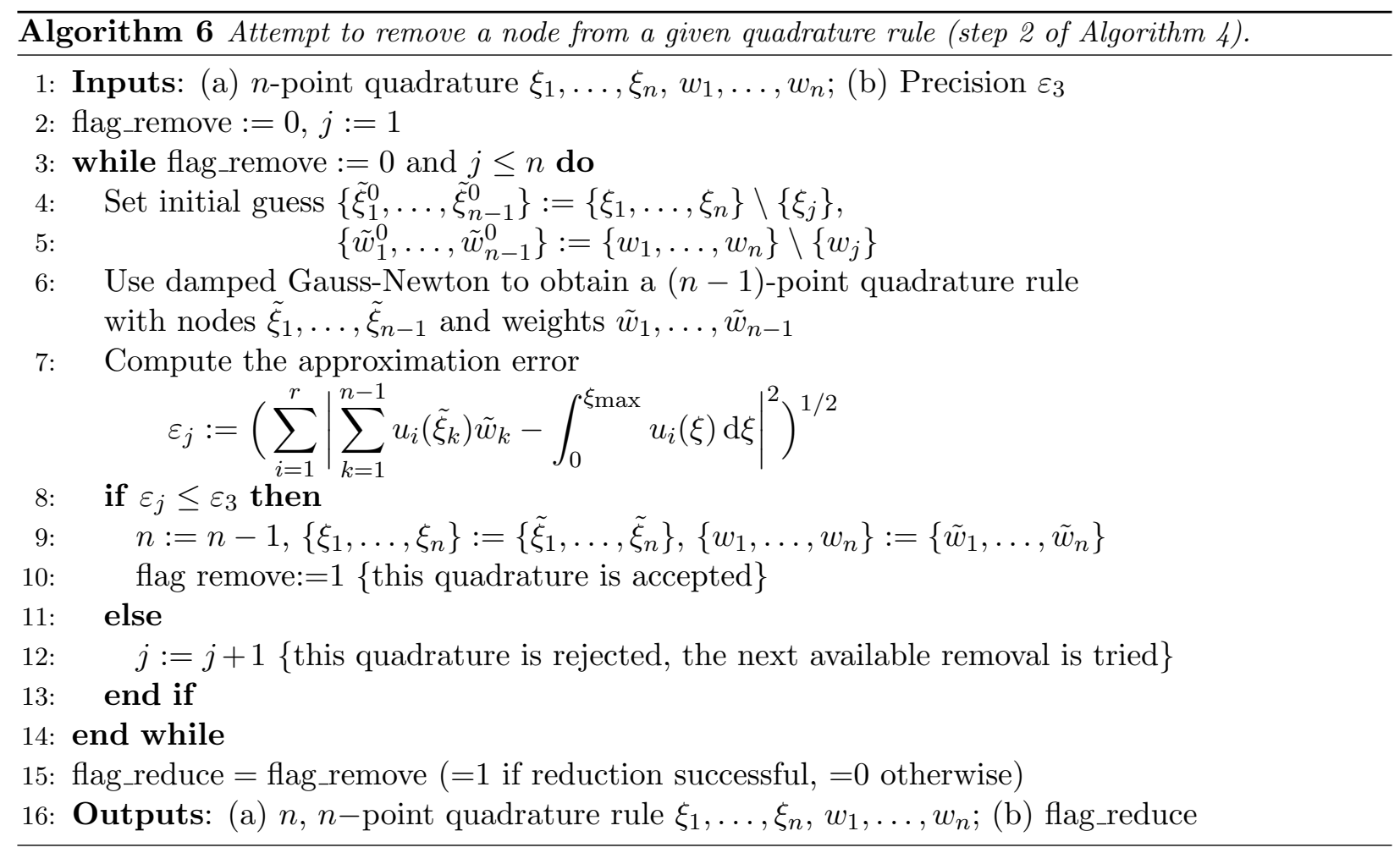




\section{$6 \quad$ Numerical tests}

In this section, computational aspects of the proposed multipole expansion of $\boldsymbol{U}_{\mathrm{C}}$ are investigated through numerical tests. The radial and angular quadrature are examined for accuracy assessment (Sec. 6.1) and empirical size adjustment (Sec. 6.2). Then, the overall complexity of the approach is estimated (Sec. 6.3). The latter task (and also, to a lesser extent, the former) is based on numerical experiments on sums of the form

$$
\boldsymbol{V}_{\mathrm{C}}[\boldsymbol{t}]:=\left\{\boldsymbol{v}[\boldsymbol{t}]^{\mathrm{T}}\left(\boldsymbol{x}_{1}\right), \ldots, \boldsymbol{v}[\boldsymbol{t}]^{\mathrm{T}}\left(\boldsymbol{x}_{M}\right)\right\}^{\mathrm{T}},
$$

with

$$
\boldsymbol{v}[\boldsymbol{t}]\left(\boldsymbol{x}_{i}\right)=\sum_{j=1}^{M} \boldsymbol{U}_{\mathrm{C}}\left(\boldsymbol{x}_{i}, \boldsymbol{x}_{j}\right) \boldsymbol{t}\left(\boldsymbol{x}_{j}\right) \quad\left(\boldsymbol{x}_{i} \in \mathcal{X}_{M}, 1 \leq i \leq M\right),
$$

where the discrete set $\mathcal{X}_{M}$ consists of $M$ regularly-spaced points located on the surface of the rectangular box defined (in terms of an arbitrary reference length $d$ ) by

$$
-4 d \leq y_{1}, y_{2} \leq 4 d, \quad-4 d \leq y_{3} \leq-d
$$

Note that the sets of evaluation and source points in (42) are taken identical merely for simplicity, as this is allowed by the non-singular nature of $\boldsymbol{U}_{\mathrm{C}}(\boldsymbol{x}, \boldsymbol{y})$ if $\boldsymbol{x}=\boldsymbol{y}$. The matrix-vector product $\boldsymbol{V}_{\mathrm{C}}[\boldsymbol{t}]$ defined by (42) is a somewhat simplified but representative (from a computational work standpoint) version of the component $\mathcal{S}_{\mathrm{C}}[\boldsymbol{t}]$ of a discretized single-layer potential (7) evaluated on a BE mesh involving $N=3 M$ DOFs. Where relevant, all the tests to follow assume material characteristics such that $\mu=3, \rho=1$ (in arbitrary units) and $\nu=0.25$; moreover, the following definition of a normalized frequency $\eta_{\mathrm{S}}$ will be used:

$$
\eta_{\mathrm{S}}=k_{\mathrm{S}} d .
$$

The GGQ-generating procedure features several tunable algorithmic parameters. The values used for the numerical experiments reported thereafter, given in Table 1, should be considered as typical (in that they allow the generation of satisfactorily-accurate GGQ rules for the present needs) rather than mandatory. The most crucial parameter is $\varepsilon_{2}$, which strongly influences the size $n_{\xi}$ and accuracy of the final GGQ rule. Moreover, the initial adaptive GaussLegendre quadrature must be highly accurate, implying a very small value for $\varepsilon_{1}$. The GGQ generation was found to be less sensitive to the remaining parameters.

\subsection{Assessment of quadrature accuracy}

Accuracy of the radial quadrature. This first set of comparisons aims at checking the accuracy of the generalized Gaussian quadrature, which is a key component of the proposed treatment. For this purpose, the integral (29) is computed with the angular integration over $\alpha$ performed analytically (for this set of tests only) in order to focus on the accuracy of the GGQ for the radial quadrature. The resulting evaluations of $\boldsymbol{U}^{\mathrm{HS}}$ are compared to corresponding values $\left(\boldsymbol{U}_{i j}^{\mathrm{HS}}\right)_{\text {ref }}$ yielded by a code (provided by B.B. Guzina [22]) which computes the elastic layered half-space

\begin{tabular}{|c|l|}
\hline Discretization of $\mathcal{P}$ & $K=30 \times 30 \times 30$ \\
\hline Algorithm 1 & $\varepsilon_{1}=10^{-14} ; \ell=20 ;$ Max \# of levels $=35$ \\
\hline Algorithm 2 & $\varepsilon_{2}=10^{-4}$ \\
\hline Algorithm 4 & $\varepsilon_{3}=10^{-8}$ \\
\hline
\end{tabular}

Table 1: Used values of the main algorithmic parameters of the GGQ-generating procedure. 
Green's tensor in non-multipole fashion (using numerical evaluation of Bessel transforms). The contributions $\boldsymbol{U}_{\infty}$ and $\overline{\boldsymbol{U}}_{\infty}$ in decomposition (8) of $\boldsymbol{U}^{\mathrm{HS}}$ are computed using the closed-form expression (13). The relative discrepancies $\varepsilon_{i j}$ on the various components, and their maximum $\bar{\varepsilon}$, are evaluated according to

$$
\varepsilon_{i j}:=\frac{\left\|\boldsymbol{U}_{i j}^{\mathrm{HS}}-\left(\boldsymbol{U}_{i j}^{\mathrm{HS}}\right)_{\mathrm{ref}}\right\|_{2}}{\left\|\left(\boldsymbol{U}_{i j}^{\mathrm{HS}}\right)_{\mathrm{ref}}\right\|_{2}}, \quad \bar{\varepsilon}:=\max _{i, j} \varepsilon_{i j} \quad(1 \leq i, j \leq 3) .
$$

While the GGQ rule was set to accurately evaluate $\boldsymbol{U}^{\mathrm{HS}}(\boldsymbol{x}, \boldsymbol{y})$ for all $(\boldsymbol{x}, \boldsymbol{y})$ such that $-4 d \leq y_{1}, y_{2} \leq$ $4 d$ and $-4 d \leq y_{3} \leq-d$, the discrepancies (45) were evaluated for a source point at $\boldsymbol{x}=(2 d, 3 d, 2 d)$ and observation points $\boldsymbol{y}$ on the line defined by $y_{1}=0.5 d, y_{2}=0.75 d$ and $-4 d \leq y_{3} \leq-d$, with the normalized frequency set to $\eta_{\mathrm{S}}=1 / \sqrt{3}$. In Table 2 , the relative discrepancies (45) on several components of $\boldsymbol{U}^{\mathrm{HS}}$ are shown for various sizes $n_{\xi}$ of the GGQ rule. As expected, the accuracy achieved on $\boldsymbol{U}^{\mathrm{HS}}$ increases with $n_{\xi}$. Importantly, all the components have a similar level of accuracy, confirming the validity of using the same quadrature for all the components.

It is also important to check whether the accuracy is influenced by the small fictitious attenuation $\gamma$ used for shifting the Rayleigh pole off the real $\xi$-axis (Sec. 4.2). Table 3 reports the discrepancy $\bar{\varepsilon}(45)$ on entries of $\boldsymbol{U}^{\mathrm{HS}}$, the rank $r$ of the set of input functions, the total number of Gauss-Legendre subintervals generated in Step 1 and the length of the smallest subinterval, for values of $\gamma$ between $10^{-3}$ and $10^{-10}$. Stable values of $\bar{\varepsilon}$ that are commensurate to the requested quadrature precision $\varepsilon_{2}$ are observed for $10^{-8} \leq \gamma \leq 10^{-5}$. Smaller values of $\gamma$ result in accuracy degradation, presumably due to the onset of numerical cancellation effects, while larger values simply distort too much the original integral. Moreover, the depth of the adaptive interval subdivision of Step 1 is, as expected, seen to increase as $\gamma$ decreases. The rank $r$ finally appears to be insensitive on $\gamma$. Note that the implementation of $\boldsymbol{U}_{\text {ref }}^{\text {HS }}$ does not depend on $\gamma$, as the frequency is kept real-valued while the Rayleigh pole is avoided by using an integration path that is deformed into the complex $\xi$-plane [22]; this makes the above comparison valid.

Accuracy of the angular quadrature. Since the integrands of the angular integrals (34) of interest are smooth periodic functions of $\alpha$, a trapezoidal rule is known to converge rapidly [12]

\begin{tabular}{|c|ccccc|}
\hline$n_{\xi}$ & $\varepsilon_{11}$ & $\varepsilon_{12}$ & $\varepsilon_{13}$ & $\varepsilon_{31}$ & $\varepsilon_{33}$ \\
\hline 12 & $4.1 \times 10^{-2}$ & $1.9 \times 10^{-2}$ & $1.5 \times 10^{-1}$ & $2.1 \times 10^{-1}$ & $2.7 \times 10^{-2}$ \\
15 & $4.4 \times 10^{-3}$ & $3.1 \times 10^{-3}$ & $2.3 \times 10^{-2}$ & $4.8 \times 10^{-2}$ & $1.8 \times 10^{-2}$ \\
16 & $1.9 \times 10^{-3}$ & $6.3 \times 10^{-4}$ & $4.8 \times 10^{-3}$ & $6.3 \times 10^{-3}$ & $1.5 \times 10^{-3}$ \\
17 & $1.2 \times 10^{-3}$ & $4.2 \times 10^{-4}$ & $3.2 \times 10^{-3}$ & $4.5 \times 10^{-3}$ & $1.4 \times 10^{-3}$ \\
23 & $5.5 \times 10^{-5}$ & $1.9 \times 10^{-4}$ & $1.7 \times 10^{-4}$ & $1.6 \times 10^{-4}$ & $1.8 \times 10^{-4}$ \\
\hline
\end{tabular}

Table 2: Relative discrepancy (45) on entries of the Green's tensor $\boldsymbol{U}^{\mathrm{HS}}$ for various values $n_{\xi}$ of the radial quadrature rule density $\left(\eta_{\mathrm{S}}=1 / \sqrt{3}\right)$.

\begin{tabular}{|c|cccccccc|}
\hline$\gamma$ & $10^{-3}$ & $10^{-4}$ & $10^{-5}$ & $10^{-6}$ & $10^{-7}$ & $10^{-8}$ & $10^{-9}$ & $10^{-10}$ \\
\hline $\bar{\varepsilon}$ & $2 \times 10^{-3}$ & $3 \times 10^{-4}$ & $2 \times 10^{-4}$ & $2 \times 10^{-4}$ & $2 \times 10^{-4}$ & $2 \times 10^{-4}$ & $5 \times 10^{-2}$ & $10^{0}$ \\
$r$ & 37 & 37 & 37 & 37 & 37 & 37 & 37 & 37 \\
\# of subintervals & 27 & 38 & 49 & 59 & 68 & 160 & 170 & 174 \\
smallest subinterval length & $2 \times 10^{-3}$ & $10^{-4}$ & $7 \times 10^{-6}$ & $9 \times 10^{-7}$ & $6 \times 10^{-8}$ & $9 \times 10^{-10}$ & $10^{-10}$ & $10^{-10}$ \\
\hline
\end{tabular}

Table 3: Maximum relative discrepancy $\bar{\varepsilon}(45)$ on entries of $\boldsymbol{U}^{\mathrm{HS}}$ for various values of the artificial attenuation $\gamma\left(\eta_{\mathrm{S}}=1 / \sqrt{3}\right)$. 


\begin{tabular}{|c|rrrrrrr|}
\hline$\xi r_{1}=\xi r_{2}$ & 1 & 5 & 10 & 15 & 20 & 25 & 30 \\
$\bar{n}_{\alpha}$ & 5 & 15 & 23 & 36 & 40 & 52 & 57 \\
\hline
\end{tabular}

Table 4: Accuracy of the angular quadrature: for each value of $\xi r_{1}$ (with $\xi r_{1}=\xi r_{2}$ here), $\bar{n}_{\alpha}$ is the smallest size $n_{\alpha}$ for which the relative error on all $I_{m, n}$ given by (34) is below $10^{-4}$.

and is used in this work:

$$
\begin{cases}\alpha_{j}=\frac{2 \pi(j-1)}{n_{\alpha}-1} & \left(1 \leq j \leq n_{\alpha}\right), \\ w_{j}^{\alpha}=\frac{2 \pi}{n_{\alpha}-1} & \left(2 \leq j \leq n_{\alpha}-1\right) \quad \text { and } \quad w_{1}^{\alpha}=w_{n_{\alpha}}^{\alpha}=\frac{\pi}{n_{\alpha}-1} .\end{cases}
$$

A quantitative illustration of the performance of this trapezoidal rule on integrals (34) is shown in Table 4 , where the smallest value $\bar{n}_{\alpha}$ of the size $n_{\alpha}$ for which the relative error on all $I_{m, n}$ defined by (34) (with respect to corresponding exact values (47)) is below $10^{-4}$ is shown for a range of values of the parameter $\xi r_{1}$ (with $\xi r_{1}=\xi r_{2}$ here). As expected, $\bar{n}_{\alpha}$ depends on the chosen value of $\xi$, see further elaboration on this issue in Sec. 6.2.

\subsection{Adjustment of the radial and angular quadrature rules}

The efficiency of the overall FMM dictates that the numbers $n_{\xi} \cdot n_{\alpha}$ of quadrature points be as small as possible. Moreover, for given clusters of source and observation points, the parameter space $\mathcal{P}$, and thus the size of the quadrature rules, depends on the frequency. Assuming a surface discretization in the form of a BE mesh (or, more generally, of sets of source and observation points lying on a surface) whose density is set, for a given configuration, so as to feature a fixed number of nodes per S-wavelength, one has $N=O\left(\eta_{\mathrm{S}}^{2}\right)$, which implies that $n_{\xi}$ and $n_{\alpha}$ depend on $N$ for a given spatial configuration.

Number of points in the generalized Gaussian quadrature. To estimate the dependency of $n_{\xi}$ on $N$, GGQ rules have been generated for several values of the frequency, such that $1 / \sqrt{3} \leq$ $\eta_{\mathrm{S}} \leq 10 / \sqrt{3}$. Table 5 shows the number $p$ of points of the piecewise-Legendre quadrature produced by Step 1 of the GGQ rule generation for this set of frequencies. As expected, large values of $p$ (ranging between 2,540 and 13,060) were obtained in all cases. Then, Fig. 3 plots the number of quadrature points $r$ reached after Step 3 (which is equal to the rank of the input functions) and the number $n_{\xi}$ of points of the final GGQ rule against $\eta_{\mathrm{S}}$. Again as expected, $r$ (and hence $n_{\xi}$ ) is much smaller than $p$, as it ranges here between 33 and 78 . While the final number of points $n_{\xi}$ is smaller than $r$, the main reduction is achieved by Step 3. Both $r$ and $n_{\xi}$ are found by curve fitting to be of order $O\left(\eta_{\mathrm{S}}^{0.3}\right)$, see Fig. 3. As a result, for surface meshes, one has the estimations $r=O\left(N^{0.15}\right)$ and $n_{\xi}=O\left(N^{0.15}\right)$.

Step 4 is claimed in [4] to reduce by about half the quadrature size (i.e. $n_{\xi} \approx r / 2$ ). This is not quite the case for the GGQ rules reported here (see Fig. 3), which might lead one to suspect a sub-optimal implementation of step 4. To clarify this, our implementation of the GGQ-generating algorithm has been also tested on other sets of input functions that are simpler (no singularity or Rayleigh pole involved) and not directly related to the present context. For example, the set of input functions (closely related to the one used in [4]) defined by

$$
\phi_{\ell}(\xi ; z)=z^{\ell} e^{\mathrm{i} \xi z} \quad\left(z:=x_{1}+\mathrm{i} x_{2}, 1 \leq x_{1}, x_{2} \leq 4,1 \leq \ell \leq 3\right),
$$

\begin{tabular}{|c|cccccccccc|}
\hline$\sqrt{3} \eta_{\mathrm{S}}$ & 1 & 2 & 3 & 4 & 5 & 6 & 7 & 8 & 9 & 10 \\
\hline$p$ & 3,360 & 5,620 & 9,560 & 13,060 & 12,680 & 12,160 & 11,660 & 12,080 & 10,560 & 7,740 \\
\hline
\end{tabular}

Table 5: Number of quadrature points $p$ obtained after Step 1 of the GGQ rule generation. 


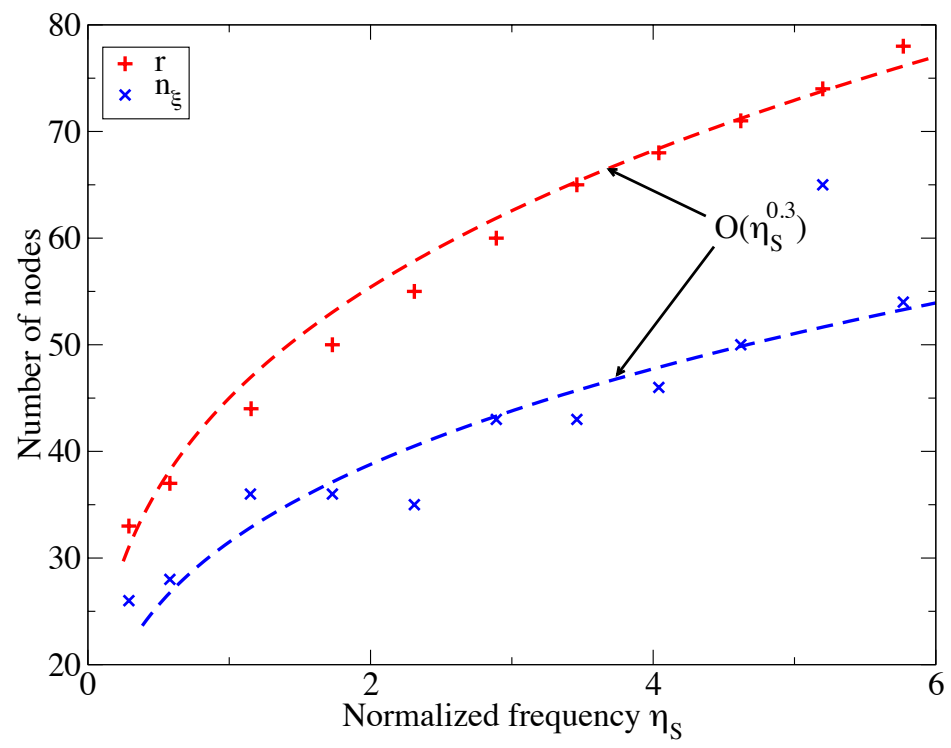

Figure 3: Rank $r$ of the input functions and number of points $n_{\xi}$ of the GGQ rule for a range of normalized frequencies $\eta_{\mathrm{S}}$.

where parameters $x_{1}, x_{2}$ belong to the same domain, and are sampled in the same way, as in the previous tests, led to GGQ rules satisfying $n_{\xi} \approx r / 2$, i.e. conforming to the behavior predicted in [4]. The efficiency of the final reduction from $r$ to $n_{\xi}$ points thus appears to depend on the properties of the chosen input functions.

Table 6 shows the relative discrepancies (45) achieved using the quadratures produced by steps 1, 3 and 4 of the GGQ generation, for three normalized frequencies. It is clear that the final GGQ rule of size $n_{\xi}$ achieves on $\boldsymbol{U}^{\mathrm{HS}}$ the same accuracy as the initial piecewise-Legendre quadrature of size $p$, despite $p$ being considerably larger than $n_{\xi}$.

Finally, one notes that as source and evaluation points are chosen closer to the free surface, $\left|x_{3}+y_{3}\right|$ decreases, making the $O\left(e^{-\xi\left|x_{3}+y_{3}\right|}\right)$ exponential decay of $\hat{\boldsymbol{U}}^{\mathrm{HS}}\left(\xi, \alpha, y_{3}\right)$ slower. This effect is expected to result in increased sizes of the radial GGQ rule. For example, considering sets of points $\chi_{M}^{a, b, c}$ such that (a) $-1.5 d \leq y_{3} \leq-0.5 d$, (b) $-1.25 d \leq y_{3} \leq-0.25 d$ or (c) $-d \leq y_{3} \leq-0.1 d$ and setting $\eta_{\mathrm{S}}=10$, the generated GGQ rules have sizes $n_{\xi}^{(a)}=53 n_{\xi}^{(b)}=82, n_{\xi}^{(c)}=144$, respectively, corroborating the expected trend.

\begin{tabular}{|c|c|ccccc|}
\hline$\eta_{\mathrm{S}}$ & Step & $\varepsilon_{11}$ & $\varepsilon_{12}$ & $\varepsilon_{13}$ & $\varepsilon_{31}$ & $\varepsilon_{33}$ \\
\hline \multirow{3}{*}{$0.5 / \sqrt{3}$} & 1 & $4.3 \times 10^{-5}$ & $2.0 \times 10^{-4}$ & $3.5 \times 10^{-4}$ & $4.9 \times 10^{-4}$ & $2.1 \times 10^{-4}$ \\
& 3 & $4.6 \times 10^{-5}$ & $2.0 \times 10^{-4}$ & $3.6 \times 10^{-4}$ & $5.1 \times 10^{-4}$ & $2.1 \times 10^{-4}$ \\
& 4 & $4.4 \times 10^{-5}$ & $2.0 \times 10^{-4}$ & $3.5 \times 10^{-4}$ & $4.9 \times 10^{-4}$ & $2.1 \times 10^{-4}$ \\
\hline \multirow{3}{*}{$5 / \sqrt{3}$} & 1 & $5.3 \times 10^{-5}$ & $1.5 \times 10^{-4}$ & $1.1 \times 10^{-4}$ & $1.3 \times 10^{-4}$ & $2.0 \times 10^{-4}$ \\
& 3 & $5.3 \times 10^{-5}$ & $1.5 \times 10^{-4}$ & $1.1 \times 10^{-4}$ & $1.3 \times 10^{-4}$ & $2.0 \times 10^{-4}$ \\
& 4 & $5.4 \times 10^{-5}$ & $1.5 \times 10^{-4}$ & $1.1 \times 10^{-4}$ & $1.3 \times 10^{-4}$ & $2.0 \times 10^{-4}$ \\
\hline \multirow{3}{*}{$10 / \sqrt{3}$} & 1 & $1.6 \times 10^{-4}$ & $8.6 \times 10^{-5}$ & $1.3 \times 10^{-4}$ & $1.5 \times 10^{-4}$ & $2.1 \times 10^{-4}$ \\
& 3 & $1.6 \times 10^{-4}$ & $8.6 \times 10^{-5}$ & $1.3 \times 10^{-4}$ & $1.5 \times 10^{-4}$ & $2.1 \times 10^{-4}$ \\
& 4 & $1.6 \times 10^{-4}$ & $8.6 \times 10^{-5}$ & $1.3 \times 10^{-4}$ & $1.5 \times 10^{-4}$ & $2.1 \times 10^{-4}$ \\
\hline
\end{tabular}

Table 6: Relative discrepancy (45) on entries of the Green's tensor $\boldsymbol{U}^{\mathrm{HS}}$ after each step of the GGQ generation, for three values of the non-dimensional frequency $\eta_{\mathrm{S}}$. 


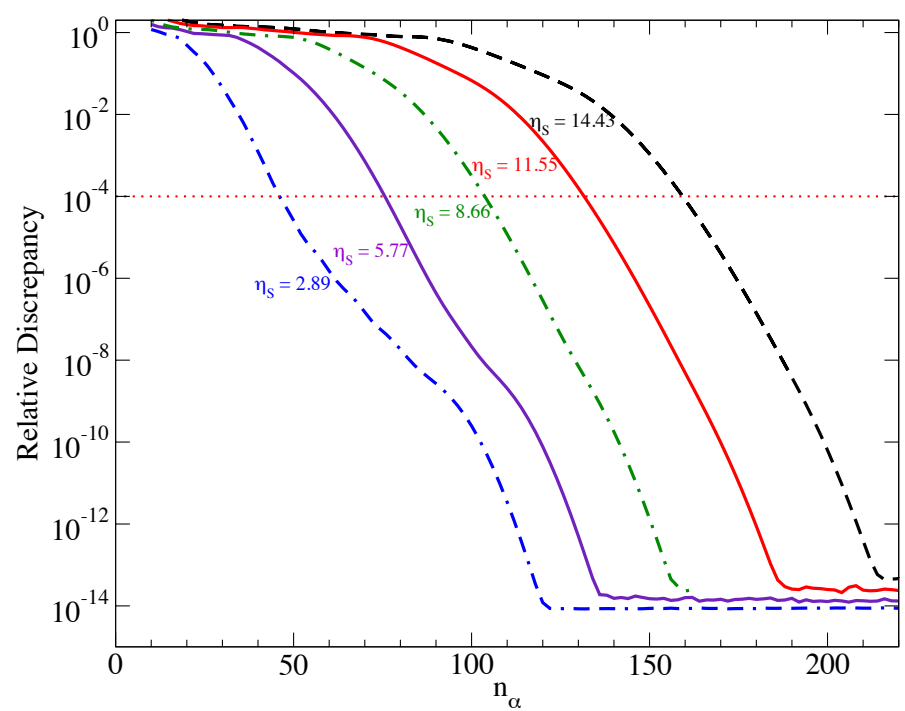

Figure 4: Relative discrepancy between computed and reference values of matrix-vector products (42) for five normalized frequencies and increasing number $n_{\alpha}$ of quadrature nodes.

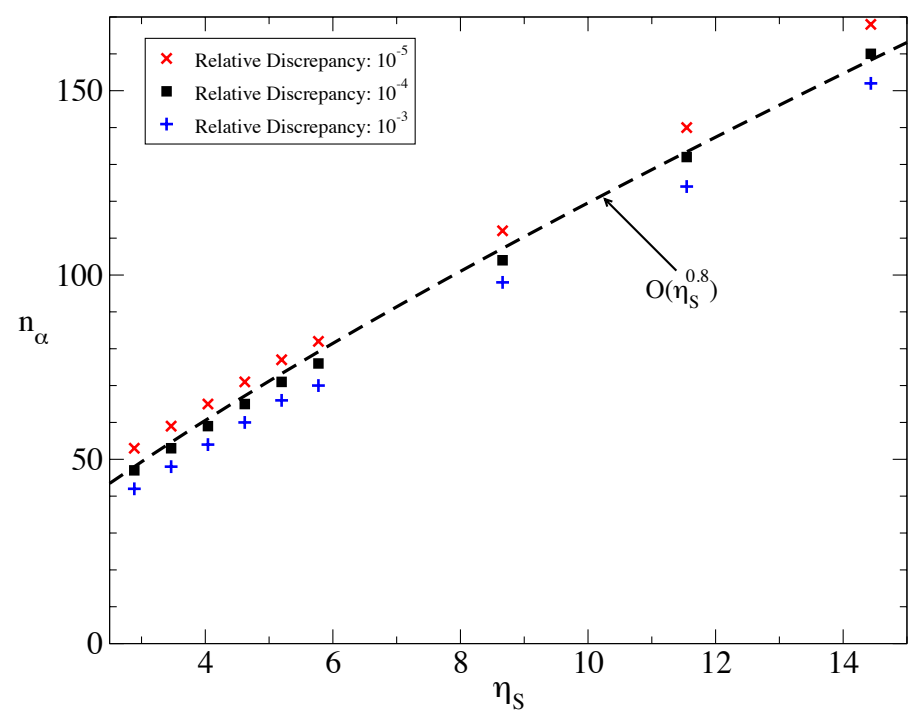

Figure 5: Number of points $n_{\alpha}$ required to achieve a relative discrepancy of $10^{-3}, 10^{-4}$ and $10^{-5}$ with the reference value of (42) against the normalized frequency.

Number of points in the angular quadrature. The oscillation in the angular integrals of (30), and hence the required size of the trapezoidal quadrature, increases with $\xi$. Here, the same trapezoidal rule is used for all $\xi$, so that $n_{\alpha}$ should a priori be chosen according to the value of $\xi_{\max }$. The latter depends in turn on the frequency through (33), which prompts one to seek a relation of the form $n_{\alpha}=f\left(\eta_{\mathrm{S}}\right)$. An empirical means towards this aim consists in evaluating the discrepancy between matrix-vector products (42) computed with the separation of variables and a reference value. Since computing the latter using the $1 \mathrm{D}$ non-multipole radial-integral representation of $\boldsymbol{U}_{\mathrm{C}}$ would be too expensive for large $N$, reference values for (42) are instead evaluated by using the separation of variables, with a large value of $n_{\alpha}$ (set to $n_{\alpha}=250$ ) to ensure accuracy. In Fig. 4 , the relative discrepancy with the reference value of (42) is represented against $n_{\alpha}$ for five normalized frequencies $\eta_{\mathrm{S}}=2.89,5.77,8.66,11.55$ and 14.43 , while Fig. 5 shows the value of $n_{\alpha}$ required to achieve a relative discrepancy of $10^{-3}, 10^{-4}$ and $10^{-5}$ for a range of frequencies. As expected, 


\begin{tabular}{|c|ccccccc|}
\hline$\sqrt{3} \eta_{\mathrm{S}}$ & 1 & 2 & 3 & 4 & 5 & 6 & 7 \\
\hline$N=3 M$ & 780 & 3696 & 8256 & 15048 & 23328 & 34452 & 46056 \\
\hline \hline$\sqrt{3} \eta_{\mathrm{S}}$ & 8 & 9 & 10 & 15 & 20 & 25 \\
\hline$N=3 M$ & & 61248 & 77028 & 96360 & 215496 & 386760 & 601704 \\
\hline
\end{tabular}

Table 7: Fast evaluation of (42): considered frequencies and corresponding DOF counts.

the value of $n_{\alpha}$ required to achieve a given accuracy increases with the frequency, with the relative discrepancy decreasing rapidly if $n_{\alpha}$ increases. To achieve a constant accuracy over the range of frequencies, $n_{\alpha}$ is found by curve-fitting to depend on the frequency according to $O\left(\eta_{\mathrm{S}}^{0.8}\right)$, implying that $n_{\alpha}=O\left(N^{0.4}\right)$. In order to achieve a good compromise between CPU time and accuracy, the setting $n_{\alpha}=\left\lfloor 18 \eta_{\mathrm{S}}^{0.8}+6\right\rfloor$ (where $\lfloor z\rfloor$ is the largest integer $\leq z$ ) is used in the following, allowing a relative precision of order $10^{-4}$ for the angular integral. Higher accuracy might be obtained by a modest increase of $n_{\alpha}$ (see Fig. 5), but $10^{-4}$ is sufficient for engineering applications. A possible optimization, not addressed here, would aim at reducing the overall angular quadrature effort by allowing $n_{\alpha}$ to depend on the location $\xi$ of a GGQ node (and hence take reduced values for small $\xi)$.

Predicted complexity for the accelerated matrix-vector product. The empirically-determined complexities of the radial and angular integrations imply that the expected overall complexity of (for example) one evaluation of an elastic potential is $O\left(N n_{\xi} n_{\alpha}\right)=O\left(N^{1.55}\right)$. Note that this estimation holds for the specific choice of spatial bounds of the clusters of source and evaluation points used for these tests, and may be affected by modifications of these bounds.

\subsection{Complexity estimation for the accelerated matrix-vector product}

Numerical verification of the overall complexity. The overall complexity of the evaluation of matrix-vector products $\boldsymbol{V}_{\mathrm{C}}[\boldsymbol{t}]$ of the form (42) is now investigated, the number and spacing of points of $\mathcal{X}_{M}$ being adjusted in order to achieve a fixed density of twenty points per S-wavelength.

To check whether its actual computation conforms with the $O\left(N^{1.55}\right)$ complexity predicted from the behavior of the quadrature rules, $\boldsymbol{V}_{\mathrm{C}}[\boldsymbol{t}]$ is computed for a range of frequencies such that $1 / \sqrt{3} \leq \eta_{\mathrm{S}} \leq 25 / \sqrt{3}$, resulting in a DOF count range $780 \leq N=3 M \leq 601,704$ (see Table 7 ). To simplify matters, a constant density such that $\boldsymbol{t}\left(\boldsymbol{x}_{i}\right)=(1,1,1)(1 \leq i \leq M)$ is used in $\boldsymbol{V}_{\mathrm{C}}[\boldsymbol{t}]$ since this choice does not affect the computational work. The matrix-vector product $\boldsymbol{V}_{\mathrm{C}}[\boldsymbol{t}]$ is then evaluated in two ways for comparison purposes, using either (i) the proposed accelerated method, or (ii) the non-accelerated method based on analytical angular integration and the radial Bessel transform (with GGQ also used for radial integration). The expected complexity of method (ii) is $O\left(n_{\xi} N^{2}\right)=O\left(N^{2.15}\right)$.

Since the complexity depends on the number of GGQ nodes, Fig. 6 shows $n_{\xi}$ as a function of $N$ for the configuration and range of frequencies considered. Curve fitting yields $n_{\xi}=O\left(N^{0.15}\right)$ as before.

In Fig. 7, the CPU times recorded for computing (42) either with acceleration (blue symbols) or without acceleration (red symbols) are plotted against the model size $N$. The proposed acceleration method is seen to drastically reduce the CPU time compared to the standard treatment of the matrix-vector product, with respective observed complexities $O\left(N^{1.55}\right)$ and $O\left(N^{2.15}\right)$ as expected (the latter method being also based on a GGQ rule for the radial Fourier-Bessel integral). The present acceleration method performs a matrix-vector product for about $N=600,000$ DOFs in about $25 \mathrm{mn}$ (on a single-processor PC), whereas the same CPU time would only allow $N \approx 10,000$ DOFs using the standard approach. 


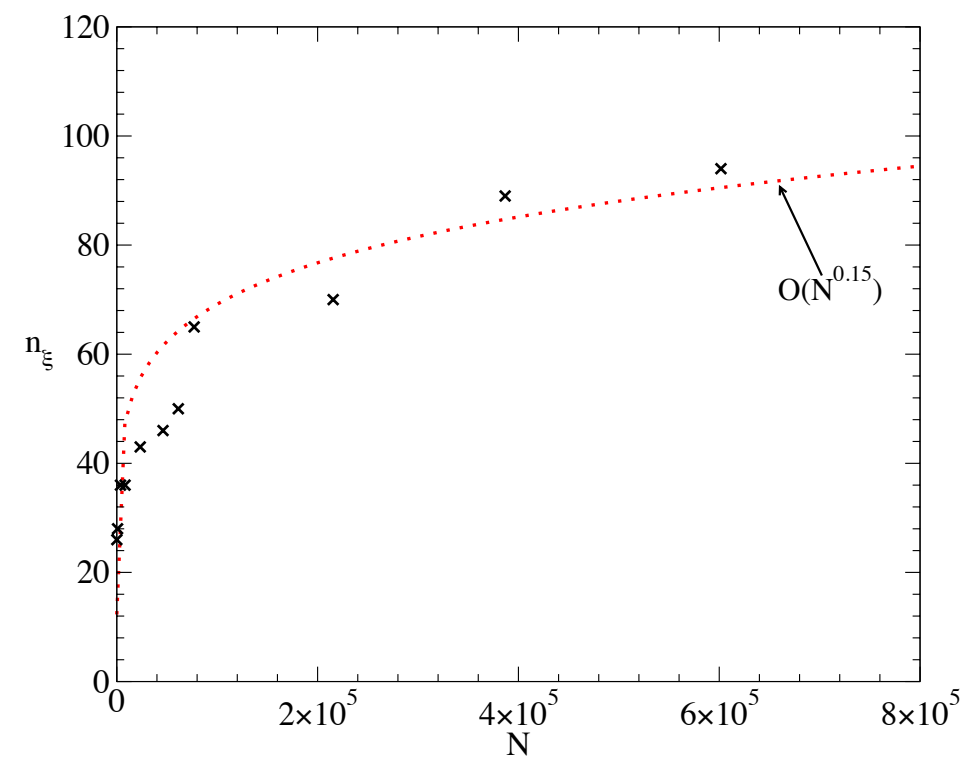

Figure 6: Fast evaluation of (42): size $n_{\xi}$ of the GGQ rule against DOF count $N$.

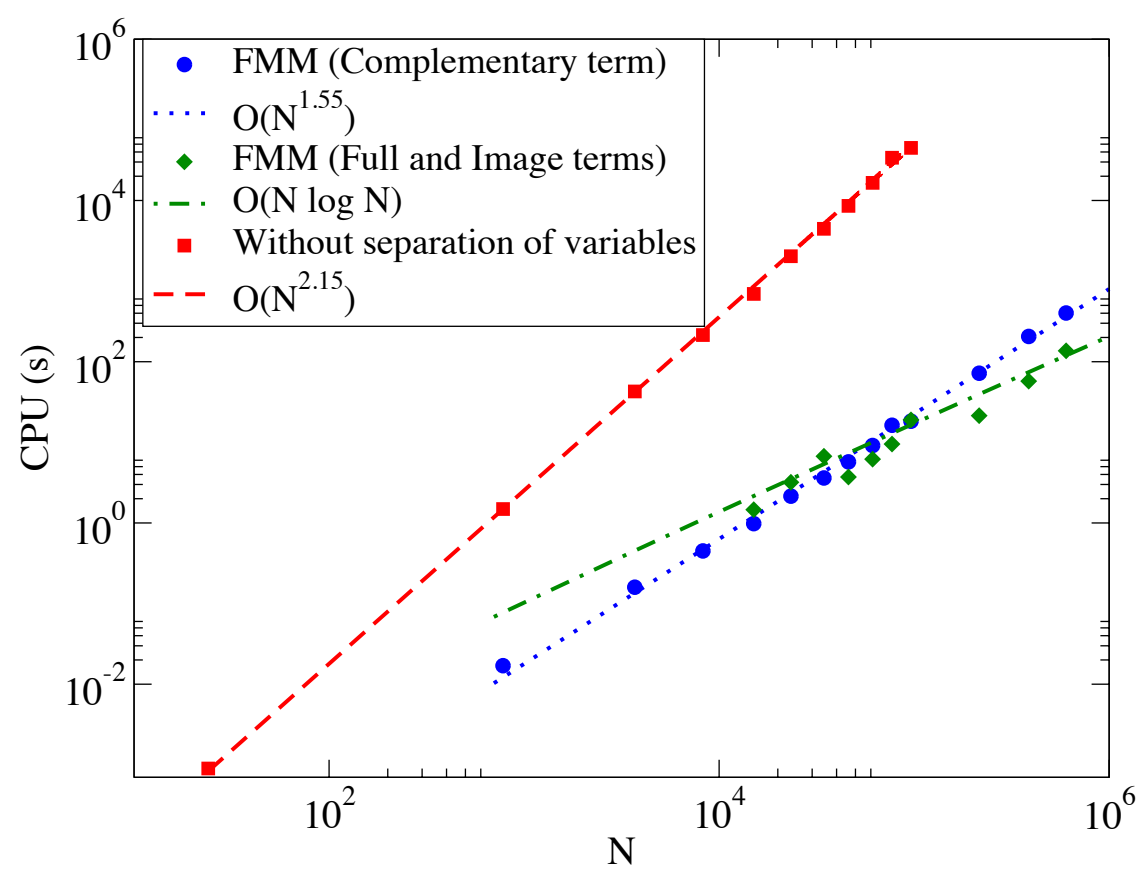

Figure 7: Numerical estimation of complexity: matrix-vector product (42) computed with acceleration (blue line and symbols) or without acceleration (red line and symbols). In addition, the complexity of the FM-accelerated matrix-vector product based on $\boldsymbol{U}_{\infty}$ and $\overline{\boldsymbol{U}}_{\infty}$ is shown (green line and symbols).

Computation of the complete half-space Green's tensor. A complete FM-BEM for halfspace problems also requires computation of the contributions involving $\boldsymbol{U}_{\infty}$ and $\overline{\boldsymbol{U}}_{\infty}$, see e.g. (10). In Fig. 7, the CPU time used in computing matrix-vector products (42) with $\boldsymbol{U}_{\mathrm{C}}$ replaced by $\boldsymbol{U}_{\infty}+$ $\overline{\boldsymbol{U}}_{\infty}$ and using the standard (diagonal-form) FMM are also shown (in green). While the complexities are different, the computing times needed for the evaluation of the complementary potential $\boldsymbol{V}_{\mathrm{C}}[\boldsymbol{t}]$ is roughly twice that required for either $\boldsymbol{V}_{\infty}[\boldsymbol{t}]$ or $\overline{\boldsymbol{V}}_{\infty}[\boldsymbol{t}]$ for the cluster sizes considered in this test.

Meshing the truncated free surface $\Gamma_{0}$ typically results in a BE model sizes 10-20 times larger than that of the meshed irregularity $S$ (for instance, a semi-spherical indentation of radius $R$ with 
a truncated free surface $\Gamma_{0}(5 R)$ [6] leads, with a uniform mesh density, to a DOF ratio of about 13). Hence a several-fold overall computational gain can be expected from using the half-space Green's tensor. Moreover, additional advantages in the proposed formulation lie in (i) substantial memory reduction, and (ii) avoidance of any loss of accuracy caused by artificial truncation.

\section{Conclusions}

The use of the elastodynamic half-space Green's tensor in the FM-BEM is a very promising avenue for enhancing the computational performances of 3D BEM applied to analyses arising from e.g. soil-structure interaction or seismology. By expressing the Green's tensor as a 2D Fourier integral (and, in particular, refraining from performing the angular integration despite the availability of exact formulae), a representation of the Green's tensor possessing the separated-variable form that permits multipole-like accelerated evaluation of elastic potentials was obtained. This is, to the authors' best knowledge, the first attempt at a fast multipole formulation of the elastodynamic half-space Green's tensor. To address the critical need of an efficient quadrature for the 2D Fourier integral, whose singular and oscillatory character precludes using usual (e.g. Gaussian) rules, generalized Gaussian quadrature rules have been used instead. The latter were generated by tailoring for the present needs the methodology of [4]. Numerical tests have been conducted to demonstrate the accuracy and numerical efficiency of the proposed FMM. In particular, a complexity significantly lower than that of the non-multipole version was shown to be achieved.

A full FM-BEM based on the proposed acceleration method for the half-space Green's tensor is currently under way. Among other things, this will permit comparisons between the accuracy of this new FM-BEM (which does not require meshing the free surface) and that of the FMBEM based on the elastic full-space Green's tensor (which requires meshing the free surface) on examples representative of applications in seismology or soil-structure interaction. On the basis of the preliminary tests presented in this article, the additional computational effort required by the evaluation of the proposed FM-compatible form of the half-space Green's tensor will be largely compensated by the computational savings resulting from the substantial reduction of the $\mathrm{BE}$ model size brought by the free surface removal, with the overall analysis time expected to be reduced several-fold. Extensions of the present method to be investigated include a fast-multipole version of the Green's tensor for the layered half-space.

Acknowledgements. The authors wish to thank Bojan B. Guzina (University of Minnesota, USA), who kindly provided his code for computing visco-elastic Green's tensor for layered halfspaces.

\section{A Closed-form expressions of angular integrals}

Closed-form expressions for integrals (34) can be derived straightforwardly from the integral representation of the Bessel function $J_{n}$, which reads

$$
J_{n}(z)=\frac{1}{\pi} \int_{0}^{\pi} \cos (z \sin \theta-n \theta) \mathrm{d} \theta
$$

This results in the following identities:

$$
\begin{array}{llrl}
I_{0,0}=2 \pi J_{0}(\xi r), & I_{1,0}=2 \pi \mathrm{i} \cos \psi J_{1}(\xi r), \\
I_{0,1}=2 \pi \mathrm{i} \sin \psi J_{1}(\xi r), & I_{2,0}=\pi J_{0}(\xi r)-\pi \cos 2 \psi J_{2}(\xi r), \\
I_{0,2}=\pi J_{0}(\xi r)+\pi \cos 2 \psi J_{2}(\xi r), & I_{1,1}=-\pi \sin 2 \psi J_{2}(\xi r) .
\end{array}
$$

\section{B Hankel transform form of $U_{\mathbf{C}}$}

The complementary part in the half-space Green's tensor is given by

$$
\boldsymbol{U}_{\mathrm{C}}(\boldsymbol{x}, \boldsymbol{y})=\int_{\mathbb{R}^{2}} e^{\mathrm{i}\left(\xi_{1}\left(y_{1}-x_{1}\right)+\xi_{2}\left(y_{2}-x_{2}\right)\right)} \hat{\boldsymbol{U}}_{\mathrm{C}}\left(\boldsymbol{\xi}, y_{3} ; \boldsymbol{x}\right) \mathrm{d} \xi_{1} \mathrm{~d} \xi_{2},
$$


with $\hat{\boldsymbol{U}}_{\mathrm{C}}$ given by (26). Upon evaluating the angular integral with the help of (46), the following expressions for the Cartesian components of $\boldsymbol{U}_{\mathrm{C}}$ are readily obtained:

$$
\begin{aligned}
\boldsymbol{U}_{\mathrm{C}}^{1 k}(\boldsymbol{x}, \boldsymbol{y}, \omega)= & \frac{1}{4 \pi \mu k_{\mathrm{S}}^{2}}\left[-\left(\delta_{1 k} \cos 2 \psi+\delta_{2 k} \sin 2 \psi\right) \int_{0}^{+\infty} A J_{2}(\xi r) \xi \mathrm{d} \xi\right. \\
& \left.+\delta_{1 k} \int_{0}^{+\infty} A J_{0}(\xi r) \xi \mathrm{d} \xi-2 \delta_{3 k} \cos \psi \int_{0}^{+\infty} B J_{1}(\xi r) \xi \mathrm{d} \xi\right], \\
\boldsymbol{U}_{\mathrm{C}}^{2 k}(\boldsymbol{x}, \boldsymbol{y}, \omega)= & \frac{1}{4 \pi \mu k_{\mathrm{S}}^{2}}\left[\left(\delta_{2 k} \cos 2 \psi-\delta_{1 k} \sin 2 \psi\right) \int_{0}^{+\infty} A J_{2}(\xi r) \xi \mathrm{d} \xi\right. \\
& \left.+\delta_{2 k} \int_{0}^{+\infty} A J_{0}(\xi r) \xi \mathrm{d} \xi-2 \delta_{3 k} \sin \psi \int_{0}^{+\infty} B J_{1}(\xi r) \xi \mathrm{d} \xi\right], \\
\boldsymbol{U}_{\mathrm{C}}^{3 k}(\boldsymbol{x}, \boldsymbol{y}, \omega)= & \frac{1}{4 \pi \mu k_{\mathrm{S}}^{2}}\left[-2\left(\delta_{1 k} \cos \psi+\delta_{2 k} \sin \psi\right) \int_{0}^{+\infty} C J_{1}(\xi r) \xi \mathrm{d} \xi\right. \\
& \left.+2 \delta_{3 k} \int_{0}^{+\infty} D J_{0}(\xi r) \xi \mathrm{d} \xi\right],
\end{aligned}
$$

where $k=1,2,3$ denotes the force direction, $\boldsymbol{y}$ and $\boldsymbol{x}$ are the source and observation locations respectively, and with the additional definitions $r:=\sqrt{\left(y_{1}-x_{1}\right)^{2}+\left(y_{2}-x_{2}\right)^{2}},\left(\frac{y_{1}-x_{1}}{r}, \frac{y_{2}-x_{2}}{r}\right)=:(\cos \psi, \sin \psi)$. Moreover, the kernel functions are defined (omitting for brevity the argument $\xi$ in $\beta, s_{\mathrm{P}}, s_{\mathrm{S}}, \delta$ ) by

$$
\begin{aligned}
& A(\xi, \boldsymbol{x}, \boldsymbol{y}, \omega)=\frac{-\xi^{2}}{\delta_{\mathrm{P}}}\left[4 s_{\mathrm{S}}^{2} s_{\mathrm{P}}^{2} e^{s_{\mathrm{S}}\left(x_{3}+y_{3}\right)}+\beta^{2} e^{s_{\mathrm{P}}\left(x_{3}+y_{3}\right)}+2 \beta s_{\mathrm{S}} s_{\mathrm{P}}\left(e^{s_{\mathrm{P}} x_{3}+s_{\mathrm{S}} y_{3}}+e^{s_{\mathrm{S}} x_{3}+s_{\mathrm{P}} y_{3}}\right)\right], \\
& B(\xi, \boldsymbol{x}, \boldsymbol{y}, \omega)=\frac{-\xi}{\delta}\left[4 \xi^{2} s_{\mathrm{S}} s_{\mathrm{P}} e^{s_{\mathrm{S}}\left(x_{3}+y_{3}\right)}+\beta^{2} e^{s_{\mathrm{P}}\left(x_{3}+y_{3}\right)}+2 s_{\mathrm{S}} s_{\mathrm{P}} \beta e^{s_{\mathrm{P}} x_{3}+s_{\mathrm{S}} y_{3}}+2 \beta \xi^{2} e^{s_{\mathrm{S}} x_{3}+s_{\mathrm{P}} y_{3}}\right], \\
& C(\xi, \boldsymbol{x}, \boldsymbol{y}, \omega)=\frac{\xi}{\delta}\left[4 \xi^{2} s_{\mathrm{S}} s_{\mathrm{P}} e^{s_{\mathrm{S}}\left(x_{3}+y_{3}\right)}+\beta^{2} e^{s_{\mathrm{P}}\left(x_{3}+y_{3}\right)}+2 \beta \xi^{2} e^{s_{\mathrm{P}} x_{3}+s_{\mathrm{S}} y_{3}}+2 \beta s_{\mathrm{S}} s_{\mathrm{P}} e^{s_{\mathrm{S}} x_{3}+s_{\mathrm{P}} y_{3}}\right], \\
& D(\xi, \boldsymbol{x}, \boldsymbol{y}, \omega)=\frac{-s_{\mathrm{P}}}{\delta}\left[4 \xi^{4} e^{s_{\mathrm{S}}\left(x_{3}+y_{3}\right)}+\beta^{2} e^{s_{\mathrm{P}}\left(x_{3}+y_{3}\right)}+2 \beta \xi^{2} e^{s_{\mathrm{P}} x_{3}+s_{\mathrm{S}} y_{3}}+2 \xi^{2} \beta e^{s_{\mathrm{S}} x_{3}+s_{\mathrm{P}} y_{3}}\right]
\end{aligned}
$$

\section{Input functions for the half-space Green's tensor}

The input functions used in Algorithm 2 to define a quadrature for (29) are given by

$$
\begin{aligned}
& f_{1}(\bar{\xi} ; \boldsymbol{p})=\frac{\bar{\xi}^{3} \bar{s}_{\mathrm{S}}^{2} \bar{s}_{\mathrm{P}}}{\bar{\delta}} \cos \left(p_{1} \bar{\xi}\right) e^{\left(p_{2}+p_{3}\right) \bar{s}_{\mathrm{S}}}, \\
& f_{2}(\bar{\xi} ; \boldsymbol{p})=\frac{\xi^{3} \bar{\beta}^{2}}{\bar{\delta} \bar{s}_{\mathrm{P}}} \cos \left(p_{1} \bar{\xi}\right) e^{\left(p_{2}+p_{3}\right) \bar{s}_{\mathrm{P}}}, \\
& f_{3}(\bar{\xi} ; \boldsymbol{p})=\frac{\xi^{3} \bar{\beta} \bar{s}_{\mathrm{S}}}{\bar{\delta}} \cos \left(p_{1} \bar{\xi}\right) e^{p_{2} \bar{s}_{\mathrm{S}}+p_{3} \bar{s}_{\mathrm{P}}}, \\
& f_{4}(\bar{\xi} ; \boldsymbol{p})=\frac{\xi^{3} \bar{s}_{\mathrm{S}}^{2} \bar{s}_{\mathrm{P}}}{\bar{\delta}} \sin \left(p_{1} \bar{\xi}\right) e^{\left(p_{2}+p_{3}\right) \bar{s}_{\mathrm{S}}}, \\
& f_{5}(\bar{\xi} ; \boldsymbol{p})=\frac{\xi^{3} \bar{\beta}^{2}}{\bar{\delta} \bar{s}_{\mathrm{P}}} \sin \left(p_{1} \bar{\xi}\right) e^{\left(p_{2}+p_{3}\right) \bar{s}_{\mathrm{P}}}, \\
& f_{6}(\bar{\xi} ; \boldsymbol{p})=\frac{\xi^{3} \bar{\beta} \bar{s}_{\mathrm{S}}}{\bar{\delta}} \sin \left(p_{1} \bar{\xi}\right) e^{p_{2} \bar{s}_{\mathrm{S}}+p_{3} \bar{s}_{\mathrm{P}}}, \\
& f_{7}(\bar{\xi} ; \boldsymbol{p})=\frac{\bar{s}_{\mathrm{P}} \xi^{5}}{\bar{\delta}} \cos \left(p_{1} \bar{\xi}\right) e^{\left(p_{2}+p_{3}\right) \bar{s}_{\mathrm{S}}}, \\
& f_{8}(\bar{\xi} ; \boldsymbol{p})=\frac{\xi \bar{s}_{\mathrm{P}} \bar{\beta}^{2}}{\bar{\delta}} \cos \left(p_{1} \bar{\xi}\right) e^{\left(p_{2}+p_{3}\right) \bar{s}_{\mathrm{P}}}, \\
& f_{9}(\bar{\xi} ; \boldsymbol{p})=\frac{\xi^{3} \bar{\beta} \bar{s}_{\mathrm{P}}}{\bar{\delta}} \cos \left(p_{1} \bar{\xi}\right) e^{p_{2} \bar{s}_{\mathrm{S}}+p_{3} \bar{s}_{\mathrm{P}}}, \\
& f_{10}(\bar{\xi} ; \boldsymbol{p})=\frac{\xi^{4} \bar{s}_{\mathrm{S}} \bar{s}_{\mathrm{P}}}{\bar{\delta}} \cos \left(p_{1} \bar{\xi}\right) e^{\left(p_{2}+p_{3}\right) \bar{s}_{\mathrm{S}}}, \\
& f_{11}(\bar{\xi} ; \boldsymbol{p})=\frac{\xi^{2} \bar{\beta}^{2}}{\bar{\delta}} \cos \left(p_{1} \bar{\xi}\right) e^{\left(p_{2}+p_{3}\right) \bar{s}_{\mathrm{P}}}, \\
& f_{12}(\bar{\xi} ; \boldsymbol{p})=\frac{\xi^{2} \bar{\beta} \bar{s}_{\mathrm{P}} \bar{s}_{\mathrm{S}}}{\bar{\delta}} \cos \left(p_{1} \bar{\xi}\right) e^{p_{2} \bar{s}_{\mathrm{S}}+p_{3} \bar{s}_{\mathrm{P}}}, \\
& f_{13}(\bar{\xi} ; \boldsymbol{p})=\frac{\bar{\beta} \xi^{4}}{\bar{\delta}} \cos \left(p_{1} \bar{\xi}\right) e^{p_{2} \bar{s}_{\mathrm{P}}+p_{3} \bar{s}_{\mathrm{S}}}, \\
& f_{14}(\bar{\xi} ; \boldsymbol{p})=\frac{\xi^{4} \bar{s}_{\mathrm{S}} \bar{s}_{\mathrm{P}}}{\bar{\delta}} \sin \left(p_{1} \bar{\xi}\right) e^{\left(p_{2}+p_{3}\right) \bar{s}_{\mathrm{S}}}, \\
& f_{15}(\bar{\xi} ; \boldsymbol{p})=\frac{\xi^{2} \bar{\beta}^{2}}{\bar{\delta}} \sin \left(p_{1} \bar{\xi}\right) e^{\left(p_{2}+p_{3}\right) \bar{s}_{\mathrm{P}}}, \\
& f_{16}(\bar{\xi} ; \boldsymbol{p})=\frac{\xi^{2} \bar{\beta} \bar{s}_{\mathrm{P}} \bar{s}_{\mathrm{S}}}{\bar{\delta}} \sin \left(p_{1} \bar{\xi}\right) e^{p_{2} \bar{s}_{\mathrm{S}}+p_{3} \bar{s}_{\mathrm{P}}}, \\
& f_{17}(\bar{\xi} ; \boldsymbol{p})=\frac{\bar{\beta} \xi^{4}}{\bar{\delta}} \sin \left(p_{1} \bar{\xi}\right) e^{p_{2} \bar{s}_{\mathrm{P}}+p_{3} \bar{s}_{\mathrm{S}}},
\end{aligned}
$$


where the non-dimensional variable $\bar{\xi}$ and functions $\bar{s}_{\mathrm{P}}, \bar{s}_{\mathrm{S}}$, and also the parameters $\boldsymbol{p}=\left(p_{1}, p_{2}, p_{3}\right)$, are as defined in the "Parameters" paragraph of Sec. 4.3, and with the additional definitions $\beta(\xi)=k_{\mathrm{S}}^{2} \bar{\beta}(\bar{\xi}), \delta(\xi)=k_{\mathrm{S}}^{4} \bar{\delta}(\bar{\xi})$.

\section{References}

[1] Aubry, D. Clouteau, D. A regularized boundary element method for stratified media. In G. Cohen, L. Halpern, P. Joly (eds.), Mathematical and numerical aspects of wave propagation phenomena, pp. 660-668. SIAM (Philadelphia, USA) (1991).

[2] Banerjee, P. K., Mamoon, S. M. A fundamental solution due to a periodic point force in the interior of an elastic half-space. Earthq. Eng. Struct. Dyn., 19:91-105 (1990).

[3] Bonnet, M. Boundary Integral Equation Method for Solids and Fluids. Wiley (1999).

[4] Bremer, J., Gimbutas, Z., Rokhlin, V. A nonlinear optimization procedure for generalized gaussian quadrature. SIAM J. Sci. Comput., 32(4):1761-1788 (2010).

[5] Çakir, Ö. The multilevel fast multipole method for forward modelling the multiply scattered seismic surface waves. Geophys. J. Int., 167:663-678 (2006).

[6] Chaillat, S., Bonnet, M., Semblat, J. F. A multi-level fast multipole BEM for 3-D elastodynamics in the frequency domain. Comput. Meth. Appl. Mech. Eng., 197:4233-4249 (2008).

[7] Cheng, H., Crutchfield, W. Y., Gimbutas, Z., Greengard, L. F., Ethridge, J. F., Huang, J., Rokhlin, V., Yarvin, N., Zhao, J. A wideband fast multipole method for the Helmholtz equation in three dimensions. J. Comp. Phys., 216:300-325 (2006).

[8] Cheng, H., Rokhlin, V., Yarvin, N. Non-linear Optimization, Quadrature and Interpolation. SIAM J. Optim., 9:901-223 (1999).

[9] Clouteau, D., Aubry, D. Computational soil-structure interaction. In W. S. Hall, G. Oliveto (eds.), Boundary element methods for soil-structure interaction, pp. 61-125. Kluwer Academic Publishers (2004).

[10] Darve, E. The Fast Multipole Method: Numerical Implementation. J. Comp. Phys., 160:195240 (2000).

[11] Darve, E., Havé, P. Efficient fast multipole method for low-frequency scattering. J. Comp. Phys., 197:341-363 (2004).

[12] Davis, P. J., Rabinowitz, P. Methods of numerical integration. Academic Press (1975).

[13] Durán, M., Godoy, E., Nédélec, J.C. Theoretical aspects and numerical computation of the time-harmonic Green's function for an isotropic elastic half-plane with an impedance boundary condition. ESAIM: Mathematical Modelling and Numerical Analysis, 44(4):671-692 (2010).

[14] Eringen, A. C., Suhubi, E. S. Elastodynamics, Vol. II-Linear Theory. Academic Press (1975).

[15] Filon, L.N.G. On a quadrature formula for trigonometric integrals. Proceedings of the Royal Society of Edeinburg, 49:38-47 (1928).

[16] Fujiwara, H. The fast multipole method for solving integral equations of three-dimensional topography and basin problems. Geophys. J. Int., 140:198-210 (2000).

[17] Gradshteyn, I. S., Ryzhik, I. M. Tables of integrals, series and products (seventh edition). Elsevier (2007). 
[18] Grasso, E., Chaillat, S., Bonnet, M., Semblat, J.F. Application of the multi-level timeharmonic fast multipole BEM to 3-D visco-elastodynamics. Eng. Anal. Bound. Elem., 36:744758 (2012).

[19] Greengard, L., Huang, J. F., Rokhlin, V., Wandzura, S. Accelerating fast multipole methods for the Helmholtz equation at low frequencies. IEEE Computational Science Engng., 5(3):3238 (1998).

[20] Guiggiani, M., Gigante, A. A general algorithm for multidimensional cauchy principal value integrals in the boundary element method. ASME J. Appl. Mech., 57:906-915 (1990).

[21] Guzina, B. B., Nintcheu Fata, S., Bonnet, M. On the stress-wave imaging of cavities in a semi-infinite solid. Int. J. Solids Struct., 40(6):1505 - 1523 (2003).

[22] Guzina, B. B., Pak, R. Y. S. On the Analysis of Wave Motions in a Multi-Layered Solid. Quart. J. Mech. Appl. Math., 54:13-37 (2001).

[23] Isakari, H., Niino, K., Yoshikawa, H., Nishimura, N. Calderon's preconditioning for periodic fast multipole method for elastodynamics in 3D. Int. J.Num. Meth. Eng., 90:484-505 (2012).

[24] Kennett, B. L. N. Seismic wave propagation in stratified media. In Advances in Applied Mechanics, vol. 21. Academic Press (1981).

[25] Kupradze, V. D. (ed.). Three-dimensional problems of the mathematical theory of elasticity and thermoelasticity. North Holland (1979).

[26] Kupradze, V. D., Gegelia, T.G. Dynamical problems in elasticity. Progress in solid mechanics, vol. III, North Holland (1963).

[27] Ma, J., Rokhlin, V., Wandzura, S. Generalized Gaussian Quadrature rules for systems of arbitrary functions. SIAM J. Numer. Anal., 33(3):971-996 (June 1996).

[28] Maurel, A., Pagneux, V., Barra, F., Lund, F. Interaction of a surface wave with a dislocation. Phys. Rev. B, 75:224112 (2007).

[29] Nishimura, N. Fast multipole accelerated boundary integral equation methods. Appl. Mech. Reviews, 55:299-324 (2002).

[30] Pak, R.Y.S., Guzina, B. B. Seismic soil-structure interaction analysis by direct boundary element methods. Int. J. Solids Struct., 36:4743-4766 (1999).

[31] Pan, L., Rizzo, F., Martin, P.A. Some efficient boundary integral strategies for time-harmonic wave problems in an elastic halfspace. Comput. Meth. Appl. Mech. Eng., 164:207-221 (1998).

[32] Rizzo, F.J., Shippy, D.J., Rezayat, M. A boundary integral equation method for time-harmonic radiation and scattering in an elastic half-space. In T.A. Cruse, A.B. Pifko, Arman. H. (eds.), Advance Topics in Boundary Element Analysis, pp. 83-90. ASME (1985).

[33] Rokhlin, V. Diagonal forms of translation operators for the Helmholtz equation in three dimensions. Applied and Computational Harmonic Analysis, 1:82-93 (1993).

[34] Saad, Y., Schultz, M. H. GMRES: a Generalized Minimal Residual Algorithm for solving nonsymmetric linear systems. SIAM Journal on Scientific Computing, 7:856-869 (1986).

[35] Takahashi, T., Nishimura, N., Kobayashi, S. A fast BIEM for three-dimensional elastodynamics in time domain. Eng. Anal. Bound. Elem., 28:165-180 (2004).

[36] Yarvin, N., Rokhlin, V. Generalized gaussian quadratures and singular value decompositions of integral operators. SIAM J. Sci. Comput., 20:699-718 (1998). 